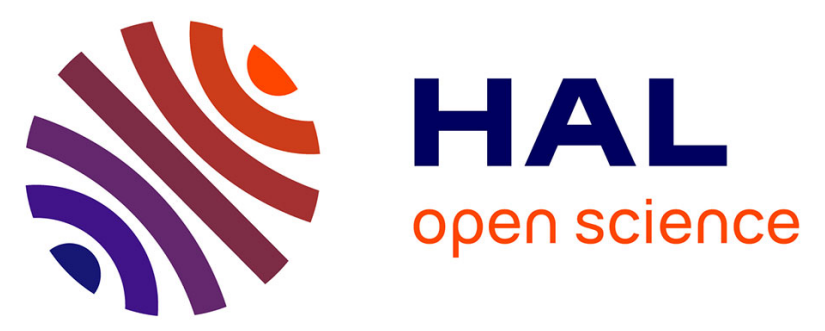

\title{
Numerical study based on the Constitutive Relation Error for identifying semi-rigid joint parameters between planar structural elements
}

\author{
Hugo Luiz Oliveira, François Louf, Fabrice Gatuingt
}

\section{To cite this version:}

Hugo Luiz Oliveira, François Louf, Fabrice Gatuingt. Numerical study based on the Constitutive Relation Error for identifying semi-rigid joint parameters between planar structural elements. Engineering Structures, 2021. hal-03173599

\author{
HAL Id: hal-03173599 \\ https://hal.science/hal-03173599
}

Submitted on 18 Mar 2021

HAL is a multi-disciplinary open access archive for the deposit and dissemination of scientific research documents, whether they are published or not. The documents may come from teaching and research institutions in France or abroad, or from public or private research centers.
L'archive ouverte pluridisciplinaire $\mathbf{H A L}$, est destinée au dépôt et à la diffusion de documents scientifiques de niveau recherche, publiés ou non, émanant des établissements d'enseignement et de recherche français ou étrangers, des laboratoires publics ou privés. 


\title{
Numerical study based on the Constitutive Relation Error for identifying semi-rigid joint parameters between planar structural elements
}

\author{
H. Oliveira, F. Louf, F. Gatuingt \\ Université Paris-Saclay, ENS Paris-Saclay, CNRS \\ LMT - Laboratoire de Mécanique et Technologie \\ 91190, Gif-sur-Yvette, France
}

\begin{abstract}
Over the past few decades, the Constitutive Relation Error (CRE) theory has proven to be a valuable numerical technique for model identification in engineering systems. Under certain conditions, this technique allows predicting the global state of connections starting from an observed structural vibratory response. These conditions often assume deterministic knowledge about input parameters and mechanical modelling. As with most inverse problems, the inherent uncertainties in the system can drastically affect the parameters of interest, posing an additional difficulty in the identification process. The present study proposes a simple and direct way to include and evaluate the effect of uncertainties in the CRE-based process of identifying parameters of semi-rigid structural connections. The numerical investigations are carried out in two parts. The first, from an exclusively deterministic perspective, shows the main characteristics of the method such as the possibility of using partial data and the inclusion of flexible support conditions. The second part explores the limits of the suggested formulation in a non-deterministic context considering both measurement noise and material uncertainty. The results show that the presented CRE formulation can identify the parameters properly in various situations. However, high noise levels in the reference measures may deteriorate identification process.
\end{abstract}

Keywords - Finite Element Model Updating, Modified Constitutive Relation Error, Planar structures, Structural joint identification

\section{Introduction}

When the term civil structure is invoked, several examples may come to mind: houses, buildings, bridges, etc. Regardless of their specific functions and complexities, they all

\footnotetext{
*Corresponding author, email: hugoitaime@gmail.com
} 
have one essential point that characterizes them and allows their systematic study: they are composed of structural elements. These fundamental units, arranged in different ways in space, generate distinct structural systems. There are three classes in which these elements can be ideally placed depending on the relative sizes of their sides: reticular (beams, columns), planar (walls, slabs), or massive (foundation blocks). This division in conceptual units, or structural elements, is advantageous for several reasons including mechanical analysis and for construction purposes. But a set of scattered structural elements does not necessarily form a structure. It is necessary to have an additional component to join these elements together. Those physical regions, where the structural elements meet, are indispensable to the system design and receive the name structural joints.

The improper functioning of the joints reflects on the performance of the structural system. This finding comes from practical nature observations. Firstly, consider the case of beams, which manifest a proximate relation to slabs in some aspects. The corresponding joints, according to its capacity to enable relative movement in a given direction, can be classified as: rigid, free, or semi-rigid. In structural analysis, it is usual considering the joints among structural members as perfectly rigid, what may not be a proper assumption in many cases. In reality, the behavior would be closer to semi-rigid [1]. Since the same practice is extensible to planar elements, it seems legitimate to put the validity of this hypothesis in question. Secondly, there is the localizing effect; shear wall/slab joints have been shown to be the area of strong deformation and degradation mechanisms. Among the reasons, one can cite the cyclic behavior of concrete under shear and the losses of bond strength under tensile and compression loads [2]. In addition, investigating these connections allows us to better understand how energy is distributed among the different elements, and how this energy mobilizes the vibration modes of each one. This information is relevant for designing more efficient structures.

Knowing the relationships between causes and effects of the various phenomena of interest to engineering has been a constant concern along the history. This effort has led to numerous effective tools, codes, practices, for the study and sizing of the various structural elements, associating safety and rational use of resources. The same remains true for structural joints. However, there are specific aspects that do not have an enough understanding and therefore inspire further investigation. The mechanical behaviour of connections between planar elements is one such example. In particular, consider the following question: how to characterize the structural joint state as realistically as possible? Being aware that various damaging phenomena start at joints [3], would it be possible to extract the essential variables that control their origin and propagation?

In the literature, it is common to find three ways to approach this subject. The first is the experimental way, in which observations show large stress concentrations and damage near the junctions arising from the strut action along the panels $[4,5,6]$. Secondly, the theoretical way, in which some hypotheses are made in order to explain the observed physical phenomena [7]. Third, the modelling way, in which computational simulations try to predict some particular aspect of the structure, such as the joint capacity [8] and the global behaviour [9]. This last way has gained prominence over the last decades because numerical simulations, alongside theory and experiment, have been consolidated as the third pillar in today's science [10]. The present study is in line with the third pillar. From the various reasons that could motivate this choice, it is highlighted those of functional 
order, for example, in nuclear power plants where intrusive measurements, or experiments, are not a simple task. In this and other similar contexts, computational models become a meaning for obtaining indirect information, provided it is able to sufficiently represent the actual structural state. It is therefore of practical importance, as shown in the state of art, to establish "best-estimate" numerical methodologies for civil structures at all levels [11]. This knowledge is of capital relevance for decision making in structural health monitoring and planning repair actions.

This brief discussion served to make clearer the choice of structural joints, and why to represent them by models. Now, it comes a crucial question: considering planar structural elements, is there an universal model to be used? In the literature, there seems to be no unanimous answer to this question [12]. In this case, there are two immediate alternatives to approach it: deriving a new model that respects the physical principles of Mechanics and reflects the observations, or, choosing a consecrated, although limited, model and adjusting its parameters to correspond some observed data sets. The latter option is known as model updating, or model identification, and is preferred here. The interest in model updating is not new and several methodologies to this end have been proposed in structural dynamics $[13,14,15]$. This effort led to the rise of two major categories of investigations. Firstly, the direct methods, in which the updating procedure does not respect physical principles necessarily [16]. Essentially, the matrices (stiffness and/or mass) are altered using exclusively the precepts of linear algebra. Second, the parametric methods, also known as indirect, iterative or local, in which the updating procedure is consistent with sound mechanical principles [17].

In the present study, the Constitutive Relation Error (CRE) [18, 19] is chosen as updating technique. In addition to being a parametric method, it has been demonstrated promising results in a number of investigations. For instance, Deraemaeker et al [20] has updated the mass and stiffness of a small scale aircraft based on modal data. They remark the high dependence on the number of degrees of freedom present on the model, which becomes prohibitive for industrial applications. In this case, they propose reduced model basis as a convenient approach to circumvent this problem [21]. In the acoustic field, CRE identification of admittance coefficient for improved simulations has led to important cost savings thanks to its good prediction of pressure levels without the need of recurrent prototyping $[22,23]$. Complex time-varying actions has been identified and compared to real in situ measures showing good agreement, which is an important step for reducing the lack of knowledge in terms of natural actions [24]. Under static loading conditions, the theoretical predictions of CRE has enabled the identification of the actual structural stiffness [25] based on experimental evidences, and also to infer the response dispersion due to random input parameters.

On its augmented version, the Modified Constitutive Relation Error (MCRE) is characterized by the adding a regularization term to the performance function. This new form broadens the possibilities of including the available information instead of making new assumptions [26, 27]. The CRE technique can also be adapted to modern experimental devices. For instance, the full-field measures provided by digital image correlation is a rich source of information for model updating [28]. Strain measures provided by fiber optic allied to damage identification is revealed to be a potential solution for civil structural health monitoring [29]. The results are consistent even in the presence of corrupted measures $[30,31]$. The stiffness identification of Euler-Bernoulli beams under static loading 
conditions has been reported in [32], as well as damage localization for a given state of the structure [33]. The use of CRE techniques in the field of reticular structural elements has been so promising that it has been possible to extend it to frame-based structures $[34,35,36,37]$. In the case of plates, the heterogeneous material properties can be identified under Reissner-Mindlin assumptions using discrete measures and few vibration modes [38]. Despite those remarkable advances in the field, the potential advantages of using CRE theory concerning civil engineering structures still deserve investigations.

In a previous work, the authors show how the deterministic formulations for parametric identification can be of theoretical and practical interest in civil engineering [39]. In the present work, we resume this deterministic formulation with straightforward modifications that allow it to be used in non-deterministic situations. The formulations, as well as the underlying assumptions, are presented throughout the Section 2. Firstly, the mathematical problem is posed on its continuous form (Section 2.1). Then, the resulting formulation is transformed into its algebraic counterpart (Section 2.2). This step is imperative for equations to be solved by using the Finite Element Method. The deterministic CRE solution algorithm is then showed in Section 2.3. In order to provide an alternative solution via, a distinct deterministic technique is presented so that it can be used to check the results provided by the CRE form (Section 2.4). Then, we suggest the modifications that may be made in the deterministic algorithm in order to include the effects of uncertainties (Section 2.5). The applications are discussed in Section 3, where the main potentialities and limitations of the proposed formulation are explored, including a discussion concerning sources of uncertainties, and how they may affect the identification process. The text ends by presenting some conclusions and suggestions in Section 4.

\section{Problem formulation}

\subsection{Continuous description}

The fundamental idea when dealing with Constitutive Relation Error theory is the separation of the governing equations into two groups. The first group contains the equations that express the conditions of admissibility: kinematic constraints and equilibrium equations. The second group contains the constitutive relations relevant to the problem. This division becomes pertinent in mechanical problems because, in general, constitutive equations are the least reliable relations. Often, relationships that describe the observed macroscopic behavior are assumed without actually having exhaustive knowledge on the micro or mesoscale. Therefore, it is natural to set these equations apart and to ensure that an approximate displacement-stress solution verifies the group of the most reliable equations (i.e. the admissibility conditions) exactly [40]. In fact, these notions can be made further precise. Let the domain of study be a deformable solid, $\Omega \subset \mathbb{R}^{3}$, with boundary, $\partial \Omega$. The structure is subjected to displacement constraints $\bar{u}$ on a part $\partial_{1} \Omega$, and traction forces $\bar{q}$ on $\partial_{2} \Omega$. The constraints are applied on complementary parts, $\partial_{1} \Omega \cup \partial_{2} \Omega=\partial \Omega$. The body force, denoted as $b$, acts through the domain. The stress tensor is represented by $\sigma$, the outward-pointing normal vector by $n$, and $\gamma$ is the acceleration's image. With these terms the core definitions can be made.

Let $(u, \sigma, \gamma)$ be a solution candidate. It is named admissible when it verifies the following three equations: 


$$
\begin{gathered}
\left.u\right|_{\partial_{1} \Omega}=\bar{u} \\
\left.\sigma n\right|_{\partial_{2} \Omega}=\bar{q} \\
\int_{\Omega} \gamma \cdot u^{*} d \Omega_{i}+\int_{\Omega} \operatorname{Tr}\left(\sigma \varepsilon^{*}\right) d \Omega=\int_{\Omega} b \cdot u^{*} d \Omega_{i}+\int_{\partial_{2} \Omega} \sigma n \cdot u_{i}^{*} d \partial \Omega, \forall u^{*} \text { with }\left.u^{*}\right|_{\partial_{1} \Omega}=0
\end{gathered}
$$

In equation (3), $\operatorname{Tr}()$ is the trace operator and $\varepsilon^{*}$ represents the symmetric part of the gradient of $u^{*}$. It is remarked that the admissibility conditions, although crucial, do not guarantee the existence and uniqueness of the solution. It is also needed to specify the constitutive relations, expressed as follows:

$$
\begin{aligned}
& f(\sigma, \varepsilon(u))=\sigma-C \varepsilon=0 \\
& g(\gamma, u)=\gamma-\rho \frac{d^{2} u}{d t^{2}}=0
\end{aligned}
$$

The equation (4) expresses a particular material response known as Hooke's Law, in which $\varepsilon$ is the linear strain tensor, and $C$ is a fourth-order stiffness tensor of material properties or Elastic moduli. In equation (5), $\rho$ is the mass density which is considered to be constant throughout the analysis.

Although it may be admissible, a triple $(u, \sigma, \gamma)$ may not satisfy exactly the constitutive relations. In this case, the relations (4) and (5) will result in nonzero values, or a residue. The quality of an admissible solution is indicated by a performance measure known as Constitutive Relation Error, $E_{C R E}^{2}$, which is a functional possessing the following two properties:

$$
\begin{gathered}
E_{C R E}^{2}(\sigma, \gamma, u) \geqslant 0 \\
E_{C R E}^{2}(\sigma, \gamma, u)=0 \Leftrightarrow(\sigma, \gamma, u) \text { is the exact solution of the problem }
\end{gathered}
$$

The way of proposing the measure is not unique and depends on the problem at hand as long as it respects the two conditions above. In the present study, the following performance measure is used:

$$
\begin{aligned}
E_{C R E}^{2}(\sigma, \gamma, u)= & \frac{\alpha}{2} \int_{\Omega} \operatorname{Tr}\left((\sigma-C \varepsilon) C^{-1}(\sigma-C \varepsilon)\right) d \Omega \\
& +\frac{(1-\alpha)}{2} \int_{\Omega}\left(\gamma-\rho \frac{d^{2} u}{d t^{2}}\right) \cdot \chi\left(\gamma-\rho \frac{d^{2} u}{d t^{2}}\right) d \Omega
\end{aligned}
$$

In equation (8), $\alpha$ is a real parameter belonging to $[0,1]$ that reflects the degree of confidence that is placed on each constitutive equation. For free-vibration problems, $\chi=\rho \omega^{2}$, where $\omega$ is the vibration frequency. Note that if the triple $(u, \sigma, \gamma)$ is admissible, and still satisfies the constitutive equations, we will have the exact solution to the problem, and the equation (8) results nil. Therefore, the solution of the mechanical problem will be the triple that minimizes equation (8), as follows: 


$$
\begin{aligned}
\min _{u, \sigma, \gamma} & E_{C R E}^{2} \\
\text { s.t. } & (u, \sigma, \gamma) \text { is admissible }
\end{aligned}
$$

This is the crucial aspect of the formulation. For a given $C$, and a given $\rho$, the formulation in equation (9) is a forward problem essentially. It is just a different way of writing the classic finite element problem. At the end, one has the structural displacements, as usual. The major advantage of this formulation is not in this direct form, but in its inverse form. The idea is finding the stiffness and mass parameters that best fit the observations. The approach consists in modifying the measure from equation (8) to include the reference quantities obtained under specific conditions [30,41]. Once these parameters are found, the model can be used to predict the structural behavior in situations different from those used for the model updating [24]. Although the static conditions can be used without major problems, here we propose the use of the dynamic data, in particular, the free vibrating regime. This choice comes from the fact that dynamic tests provide rich information about the structural state. The reference quantities are included in the equation (8) giving rise to a modified performance measure. This new version receives the name Modified Constitutive Relation Error (MCRE), and is written as follows:

$$
E_{M C R E}^{2}(\sigma, \gamma, u)=E_{C R E}^{2}(\sigma, \gamma, u)+\frac{r}{2(1-r)}\|u-\tilde{u}\|^{2}
$$

where $\tilde{u}$ represents observed displacements and $r$ is a real parameter belonging to $[0,1]$ which controls the level of confidence agreed to the measurements.

For a given $C, \rho$ and $\tilde{u}$ the equation (10) provides a positive scalar. The lower this value, the better the solution represents the observed reality. The term better means that the found solution respects strictly the admissibility conditions, and, simultaneously, it respects the stipulated constitutive equations and the observed measures as well as possible. In this case, the final problem is expressed by a double minimization which can be written as follows:

$$
\begin{aligned}
\min _{C, \rho} \min _{u, \sigma, \gamma} & E_{M C R E}^{2} \\
\text { s.t. } & (u, \sigma, \gamma) \text { is admissible }
\end{aligned}
$$

In addition to the usual information such as displacements, stresses or deformations, the solution of problem (11) provides the parameters $C$ and $\rho$ that best represent the current state of the structure. This idea remains valid for the entire structure, including the joints. In fact, this formulation even permits one can simply choose the regions that should be updated, and thus obtain the desired information. This process can be done in the discretization step and will be explained in the sequence.

\subsection{Algebraic description}

Only a few problems have a known analytical solution. This is why approximate numerical solutions have gained attention in the recent decades. Among these methods of approximation, one of th most often used in the practical engineering problems is the Finite Element Method (FEM) [42]. This method consists of subdividing the structure 
into a set of elements and nodes composing the mesh. Thus, the equilibrium equation (3) can be rewritten as follows:

$$
[K]\{u\}+[M]\{\ddot{u}\}=\{q\}
$$

where $[K]$ is the stiffness matrix, $[M]$ is the mass matrix, $\{u\}$ is the nodal displacements, $\{\ddot{u}\}$ is the nodal acceleration, and $\{q\}$ is the nodal excitation forces. Under the assumption that both displacement and excitation force are sinusoidal functions of time, it follows:

$$
\begin{aligned}
& \{u\}=\left\{A_{u}\right\} \sin \omega \tau \\
& \{q\}=\left\{A_{q}\right\} \sin \omega \tau
\end{aligned}
$$

where $\left\{A_{u}\right\}$ is the nodal displacement amplitude, $\left\{A_{q}\right\}$ is the nodal force amplitude and $\tau$ is the time. Substituting equations (13) and (14) into equation (12), the discrete equilibrium equation, valid for undamped systems, becomes:

$$
\left([K]-\omega^{2}[M]\right)\left\{A_{u}\right\}=\left\{A_{q}\right\}
$$

The present study is devoted to free vibration regimes. In this case, $\left\{A_{q}\right\}$ is nil and the equilibrium of the structure can be achieved at well-defined vibration frequencies, called proper frequencies, or eigenfrequencies. Each eigenvalue $\omega_{i}$ corresponds to a geometric shape obtained from the eigenvector, $\{U\}_{i}$. Both eigenvalues and eigenvectors are characteristic of each structure, and can be found by solving the following equation:

$$
[K]\{U\}_{i}-\omega_{i}^{2}[M]\{U\}_{i}=\{0\}
$$

Before proceeding with discretization, it is necessary to define the norm $\|u-\tilde{u}\|$ in equation (10). This norm expresses the discrepancy between numerical model predictions and observed measurements and can be proposed in multiple ways. Here, it is set the following form:

$$
\|u-\tilde{u}\|=\{[\Upsilon] U-\tilde{U}\}^{T}\left[G_{u}\right]\{[\Upsilon] U-\tilde{U}\}
$$

where $[\Upsilon]$ is a projection matrix, $\tilde{U}$ stores the collected measures and $\left[G_{u}\right]$ is defined as the stiffness matrix condensed at the points where the displacement field is being measured.

In the present study, it is assumed that the causes of the discrepancies between model and reality lie in the lack of knowledge of the correct stiffness of the system, in particular, the joints. Therefore, the mass distribution of the system is assumed perfectly known. To implement this option, we simply set $\alpha=1$ in equation (8). Let $\{V\}$ represent the image of the admissible stress. Then, the performance measure stipulated by equation (8) can be written as follows:

$$
\bar{E}_{C R E}^{2}(U, V, \theta)=\frac{1}{2}\{U-V\}^{T}[K(\theta)]\{U-V\}
$$

where $\theta$ stores the material constitutive parameters. Here, the constitutive material is linear elastic and isotropic, therefore, defined by the Poisson's ratio and the Young modulus. 
Structural joints are considered independently of the rest of the structure. In this case, each joint is considered to have semi-rigid behavior governed by Hooke's law. This choice does not imply loss of generality in terms of choosing more complex constitutive models. On the contrary, the reasons are twofold. Firstly, it allows for a clearer and more concise presentation of the underlying formulation. Second, once verified, this formulation can serve as a first approximation for more complex models (linearization step). As an independent entity, each hookean joint has stiffness according to the six possible degrees of freedom, being three rotations and three translations. The set of all stiffness parameters that characterize each joint is included in $\theta$.

The present CRE formulation allows identification to be performed only in some regions of interest. For this, it is necessary to select these regions using projection matrices on the nodal displacements in equation (18). Let $[\Pi]$ be the projection matrix which collects the degrees of freedom corresponding to the joints. It is also important to collect the stiffness matrix corresponding exclusively to the joints, i.e., $\left[K_{J}\right]$. Then, the CRE norm is rewritten as follows:

$$
\bar{E}_{C R E}^{2}(U, V, \theta)=\frac{1}{2}\{[\Pi](U-V)\}^{T}\left[K_{J}(\theta)\right]\{[\Pi](U-V)\}
$$

Using equations (17) and (18), it is possible to express equation (10) in discrete version as:

$$
\begin{aligned}
\bar{E}_{M C R E}^{2}(U, V, \theta)= & \frac{1}{2}\{[\Pi](U-V)\}^{T}\left[K_{J}(\theta)\right]\{[\Pi](U-V)\} \\
& +\frac{1}{2} \frac{r}{1-r}\{[\Upsilon] U-\tilde{U}\}^{T}\left[G_{u}\right]\{[\Upsilon] U-\tilde{U}\}
\end{aligned}
$$

The final version of the problem to be solved is then stated as follows:

$$
\begin{aligned}
\min _{\theta} \min _{U, V} & \bar{E}_{M C R E}^{2} \\
\text { s.t. } & {[K]\{V\}-\omega^{2}[M]\{U\}=\{0\} }
\end{aligned}
$$

At this point, one can choose the most convenient alternative to solve this minimization problem. Here, we choose a two-step approach as described below.

\subsection{Proposed algorithm}

The double minimization expressed in equation (21) has an appealing aspect. For a fixed $\theta$, the quantity to be minimized is quadratic into respect to $\{U-V\}$. This observation allows the unknown nodal values to be obtained directly. Let the problem expressed in equation (21) be rewritten as an unconstrained minimization using the following Lagrangian form:

$$
\begin{aligned}
L(U, V, \theta, \lambda)= & \frac{1}{2}\{[\Pi](U-V)\}^{T}\left[K_{J}(\theta)\right]\{[\Pi](U-V)\} \\
& +\frac{1}{2} \frac{r}{1-r}\{[\Upsilon] U-\tilde{U}\}^{T}\left[G_{u}\right]\{[\Upsilon] U-\tilde{U}\}+\{\lambda\}^{T}\left([K(\theta)]\{V\}-\omega^{2}[M]\{U\}\right)
\end{aligned}
$$


For a given set of parameters, $\theta^{f}$, the Lagrangian becomes function of $U, V$ and $\lambda$. Therefore, its minimum is obtained by doing:

$$
\begin{aligned}
& \frac{\partial L}{\partial U}=[\Pi]^{T}\left[K_{J}\left(\theta^{f}\right)\right][\Pi]\{U-V\}+\frac{r}{1-r}\left[G_{u}\right]\{[\Upsilon] U-\tilde{U}\}-\omega^{2}[M]\{\lambda\}=\{0\} \\
& \frac{\partial L}{\partial V}=[\Pi]^{T}\left[K_{J}\left(\theta^{f}\right)\right][\Pi]\{U-V\}+\left[K\left(\theta^{f}\right)\right]\{\lambda\}=\{0\} \\
& \frac{\partial L}{\partial \lambda}=\left[K\left(\theta^{f}\right)\right]\{V\}-\omega^{2}[M]\{U\}=\{0\}
\end{aligned}
$$

These equations can be arranged in a linear system form:

$$
[A]\{X\}=\{B\}
$$

where,

$$
\begin{gathered}
{[A]=\left[\begin{array}{ccc}
{\left[J\left(\theta^{f}\right)\right]} & \frac{r}{1-r}\left[G_{u}\right][\Upsilon] & -\omega^{2}[M] \\
{\left[J\left(\theta^{f}\right)\right]} & 0 & {\left[K\left(\theta^{f}\right)\right]} \\
-\left[K\left(\theta^{f}\right)\right] & {\left[K\left(\theta^{f}\right)\right]-\omega^{2}[M]} & 0
\end{array}\right]} \\
{\left[J\left(\theta^{f}\right)\right]=[\Pi]^{T}\left[K_{J}\left(\theta^{f}\right)\right][\Pi]} \\
\{B\}=\left\{\begin{array}{c}
\frac{r}{1-r}\left[G_{u}\right] \tilde{U} \\
0 \\
0
\end{array}\right\} \\
\{X\}=\left\{\begin{array}{c}
U-V \\
U \\
\lambda
\end{array}\right\}
\end{gathered}
$$

Both the matrices $[J]$ and $[K]$ depend on $\theta^{f}$. This implies that the solution of the linear system $(\{U\},\{V\},\{\lambda\})$ is also a function of $\theta^{f}$. This fact can be used to obtain the sensitivity of the Lagrangean at this particular point for a particular $\omega$. The derivation follows:

$$
\begin{gathered}
\nabla_{i}=\frac{\partial}{\partial \theta_{i}} L(U(\theta), V(\theta), \lambda(\theta), \theta) \\
\nabla_{i}=\underbrace{\frac{\partial L}{\partial U}}_{=0} \frac{\partial U}{\partial \theta_{i}}+\underbrace{\frac{\partial L}{\partial V}}_{=0} \frac{\partial V}{\partial \theta_{i}}+\underbrace{\frac{\partial L}{\partial \lambda}}_{=0} \frac{\partial \lambda}{\partial \theta_{i}}+\frac{\partial L}{\partial \theta_{i}}
\end{gathered}
$$

The quantities $\frac{\partial L}{\partial U}, \frac{\partial L}{\partial V}$ and $\frac{\partial L}{\partial \lambda}$ are null because $\{U\},\{V\},\{\lambda\}$ come from the Lagrangian stationariness. It comes:

$$
\nabla_{i}=\frac{\partial L}{\partial \theta_{i}}
$$




$$
\begin{aligned}
\nabla_{i} & =\frac{\partial}{\partial \theta_{i}}\left[\frac{1}{2}\{[\Pi](U-V)\}^{T}\left[K_{J}(\theta)\right]\{[\Pi](U-V)\}+\frac{1}{2} \frac{r}{1-r}\{[\Upsilon] U-\tilde{U}\}^{T}\left[G_{u}\right]\{[\Upsilon] U-\tilde{U}\}\right. \\
& \left.+\{\lambda\}^{T}\left([K(\theta)]\{V\}-\omega^{2}[M]\{U\}\right)\right]
\end{aligned}
$$

$$
\nabla_{i}=\frac{1}{2}\{U-V\}^{T}[\Pi]^{T} \frac{\partial\left[K_{J}\right]}{\partial \theta_{i}}[\Pi]\{U-V\}-\{\lambda\}^{T} \frac{\partial[K]}{\partial \theta_{i}}\{V\}
$$

From an initial value of $\theta^{f}$, and with the gradient of expression (35), it is possible to obtain an improved value $\theta^{f+1}$ using the classical steepest gradient method. With the new $\theta^{f+1}$, the procedure is repeated iteratively until convergence is reached, which concludes the solution of the problem (21). For improving computational efficiency, it is advised the use of more advanced algorithms for this purpose, such as the BFGS. A flowchart containing the steps to be followed is shown in the Figure 1. This sequence of tasks can be implemented in any finite element analysis software provided that one can manipulate the main matrices freely. In the present study, we chose to build the algorithm using Cast3m [43].

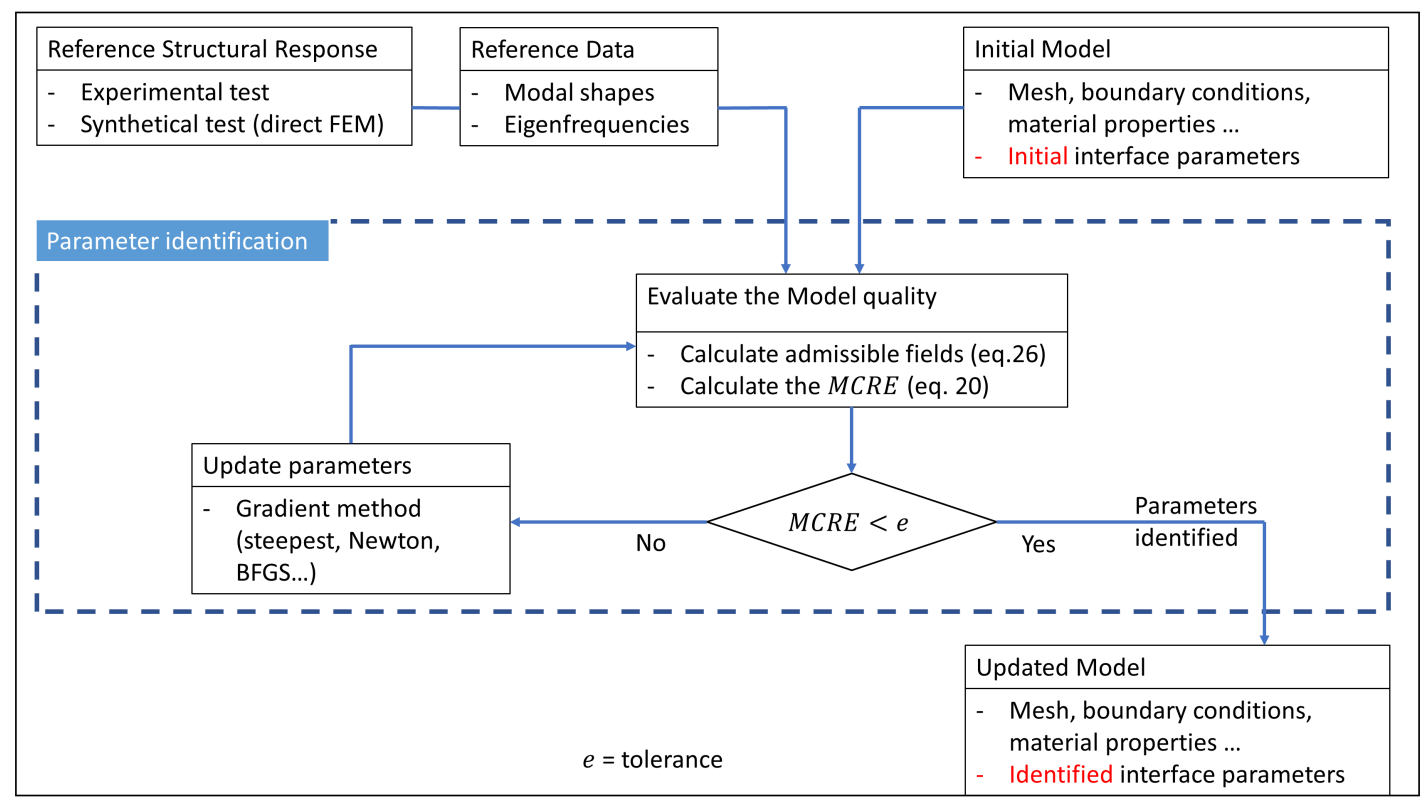

Figure 1: Minimization: proposed workflow

\subsection{The consistency measure}

The degree of consistency between the measured vibration modes and those predicted by the discrete model is indicated by the Modal Assurance Criterion (MAC). This quantity is calculated as follows: 


$$
\operatorname{MAC}\left(\tilde{U}_{i}, U_{j}\right)=\frac{\left(\{\tilde{U}\}_{i}^{T}\{U\}_{j}\right)^{2}}{\left(\{\tilde{U}\}_{i}^{T}\{\tilde{U}\}_{i}\right)\left(\{U\}_{j}^{T}\{U\}_{j}\right)}
$$

The MAC results a real number ranging from 0 to 1 . Also, the degree of consistency can be defined for the frequencies accordingly:

$$
\Delta f\left(\tilde{\omega}_{i}, \omega_{j}\right)=\frac{\tilde{\omega}_{i}-\omega_{j}}{\tilde{\omega}_{i}}
$$

The quantity $\Delta f$ can assume negative values. If the measured frequency is close enough to the corresponding predicted value, then $\Delta f$ tends to zero.

Equations (36) and (37) can be used to create an alternative expression that measures the discrepancy between observed and expected behaviour under free vibration regime. Let $h_{i}$ be a weighting factor, then, the following measure is presented:

$$
E_{M A C}^{2}=\sum_{i=1}^{N m} h_{i}\left[1-\operatorname{MAC}\left(\tilde{U}_{i}, U_{i}\right)\right]^{2}+\sum_{i=1}^{N m}\left(1-h_{i}\right)\left[\Delta f\left(\tilde{\omega}_{i}, \omega_{i}\right)\right]^{2}
$$

where $N_{m}$ represents the number of observed modes. The quantity $E_{M A C}^{2}$ may be used for verifying purposes, as will be discussed.

\subsection{Uncertainties modelling}

In the formulations described above, a perfect knowledge of both the measurements and the material parameters that constitute the planar structural elements is implicit. In fact, this hypothesis has a relevant aspect both from a theoretical point of view and for preliminary investigations [39]. However, reality requires a greater degree of sophistication on the part of models. This is due to the numerous intrinsic sources of uncertainties, such as, those regarding the physical parameters, the chosen model, the solution method employed, the accuracy achieved, the degree of knowledge about the physical phenomenon, the measurement collection, among others. Uncertainties are pervasive, to a greater or lesser extent. Thus, before pointing out whether the identified parameters correspond to reality, it is important to estimate the impact that uncertainties may have on the proposed identification strategy. In the present study, this estimate will be obtained relying on the probability theory.

Let the uncertainties in the input parameters be represented by $\boldsymbol{\Psi}=\left(\Psi_{1}, \Psi_{2}, \ldots, \Psi_{n}\right)$, a vector containing $n$ random variables. Because $\boldsymbol{\Psi}$ is known a priori, so does its cumulative probability functions, $F_{\Psi_{i}} . \psi_{j}$ is the realization of the $j$-th random variable which can follow several models of continuous distribution. This choice does not change the essence of the methodology. Therefore, for sake of simplicity, the random variables are assumed as Gaussian, being defined by two real parameters each: the mean, $m_{E}$, and the standard deviation, $s_{D}$.

Probabilistic analysis usually require several calls of the mechanical model in question to obtain the desired distributions. Here, each call represents a double minimization problem (see Figure 1). Therefore, the time spent for analysis can reach weeks, and even months, what quickly becomes a limiting factor. To circumvent this problem, it is possible 
to use a meta-modelling technique known as the Response Surface (RS). In practice, this method is a non linear regression that has been improved since its proposition [44]. In the present study, the quadratic surface response is chosen according to the following equation:

$$
\phi=a_{0}+\sum_{j=1}^{n} a_{j} \psi_{j}+\sum_{j \leq l=1}^{n} a_{j l} \psi_{j} \psi_{l}+\epsilon
$$

In equation $(39), a_{j},(j=0, \ldots, n)$ and $a_{j l},(j \leq l=1, \ldots, n)$ are real coefficients to be determined from numerical experiments by using non linear regression. $\epsilon$ represents the deviation comparing with the actual mechanical response. It follows Gaussian distribution with zero mean. The numerical experiments utilize elementary statistical information on the basic variables (mean values and standard deviations). The experimental points are generated using the following rule [45]:

$$
\psi_{j}=m_{E j}+\delta_{j} s_{D j}
$$

where $\delta_{j}$ is real parameter to set the distance from the mean, $\delta_{j} \in[-1,1]$.

With these definitions, based on the Monte Carlo simulation method, it is possible to obtain an estimate of the effects of uncertainties on the proposed formulation using the Algorithm 1.

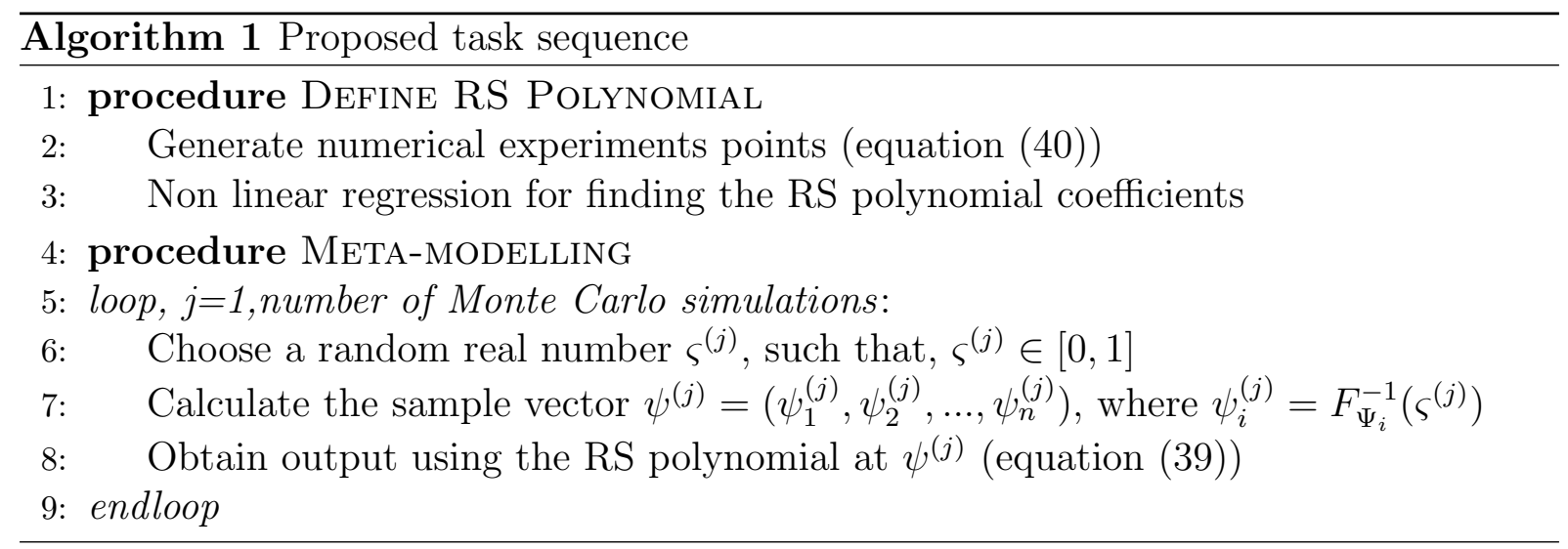

\section{Applications}

The present applications illustrate some features of the proposed formulation. We start with a simple one-dimensional structure in which some key ideas are discussed. We then move to a beam model, well known in the literature, as a means of verification and comparison with other model update techniques. Finally, in a more elaborate example, the effects of uncertainties on the identification process will be analysed relying on a structure of a building composed of planar elements.

\subsection{Two-bar structure}

Consider an ideal structure composed of two bars as illustrated in Figure 2. The bars are joined by a spring. The left end of the first bar is fixed and the right end of the second 
bar is free. Each bar has input data $E=1, S=1, L=1$ and mass per unit volume $\rho=2$. The units are assumed to be compatible for facilitating the presentation. The discrete model is represented by two standard bar elements.

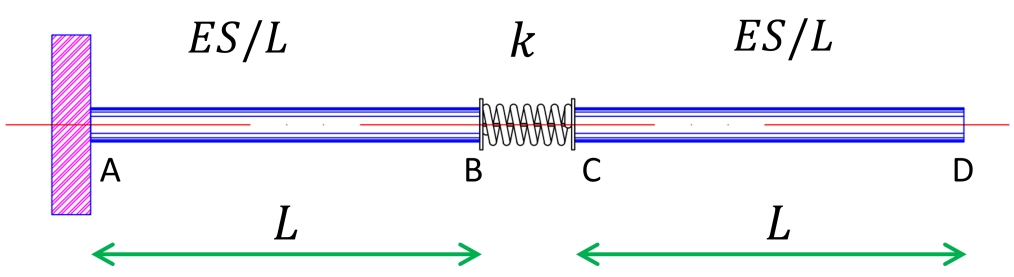

Figure 2: Two-bar structure

The reference nodal displacements for this system are showed in table 1 and the corresponding mode frequencies in table 2. They were obtained by solving the standard free vibration FEM problem using the reference value $k=2$ for the spring and the variational consistent mass matrix.

\begin{tabular}{clll}
\hline Position & Mode 1 & Mode 2 & Mode 3 \\
\hline$A$ & 0.0000 & 0.0000 & 0.0000 \\
$B$ & 0.3946 & -0.7442 & 0.3465 \\
$C$ & 0.5585 & -0.3216 & -0.7556 \\
$D$ & 0.7296 & 0.5854 & 0.5559 \\
\hline
\end{tabular}

Table 1: Normalized reference mode shapes

\begin{tabular}{ll}
\hline Eigenfrequency & Value $(\mathrm{Hz})$ \\
\hline 1 & 0.0803 \\
2 & 0.2849 \\
3 & 0.5289 \\
\hline
\end{tabular}

Table 2: Reference frequencies

The evolution of the MCRE as a function of the spring stiffness is shown in Figure 3. There are two aspects that deserve to be discussed by observing this figure. The first one is the point of minimum. According to the formulation expressed in equations (11) and (21), the searched parameter corresponds to the minimum. In this particular problem, the parameter to be identified is unique, $k$. Each point in the curve from Figure 3 is obtained from the solution of the linear system (26). However, only at the minimum point, we have a pair of admissible vectors $U$ and $V$ in which the interface stiffness parameter respects the constitutive equation as best as possible. It is important to note that we are dealing with an ideal example; there is no uncertainty in the measurements, and therefore the constitutive equations of the model is satisfied exactly. This is why the MCRE norm 
tends to zero in the neighbourhood of $k=2$. In realistic cases, MCRE null values are unlikely to occur.

The second aspect of interest of the Figure 3 is the convex behavior in the vicinity of the minimum point. This property is of considerable interest from a numerical point of view. First because it ensures the existence of local minimums contributing to the stability of the algorithm. Second, one can take advantage of second-order minimization strategies to improve computational efficiency. Of course, this idea remains valid as long as these properties are not influenced by data noise.

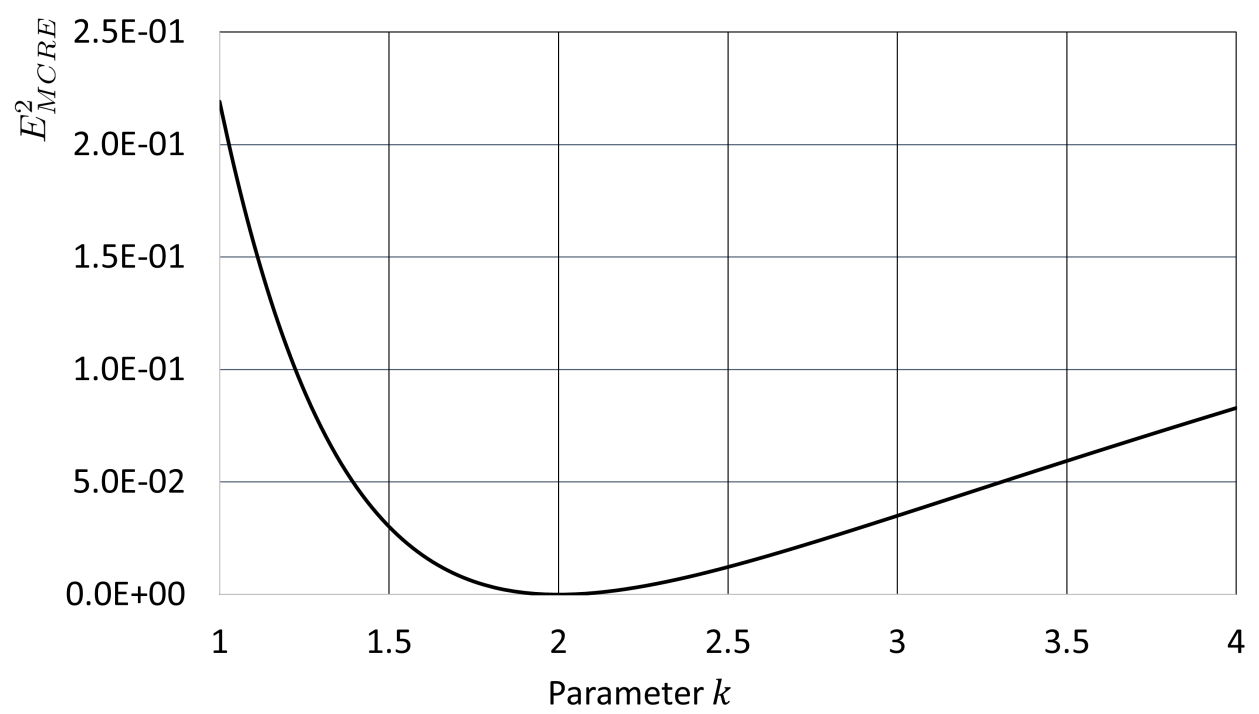

Figure 3: Two-bar structure: Evolution of Modified Constitutive Relation Error

Continuing the investigations, in free vibration regimes, it is important to observe the structural behavior over a defined frequency spectrum, and not only a specific mode. In figure 4 , it is showed the frequency response function (FRF) observed at point D (Figure 2) in terms of displacements. The frequency domain ranges from $0 \mathrm{~Hz}$ to $1.5 \mathrm{~Hz}$, containing the three eigenfrequencies. One can see the response spectrum of the system when the initial value of the searched parameter is $k=10$. After convergence, the response matches the reference. Since the reference data is ideal, and therefore free of noise, the formulation is still able to identify it appropriately, even when the initial value of the searched parameter is five times higher than the expected one. 


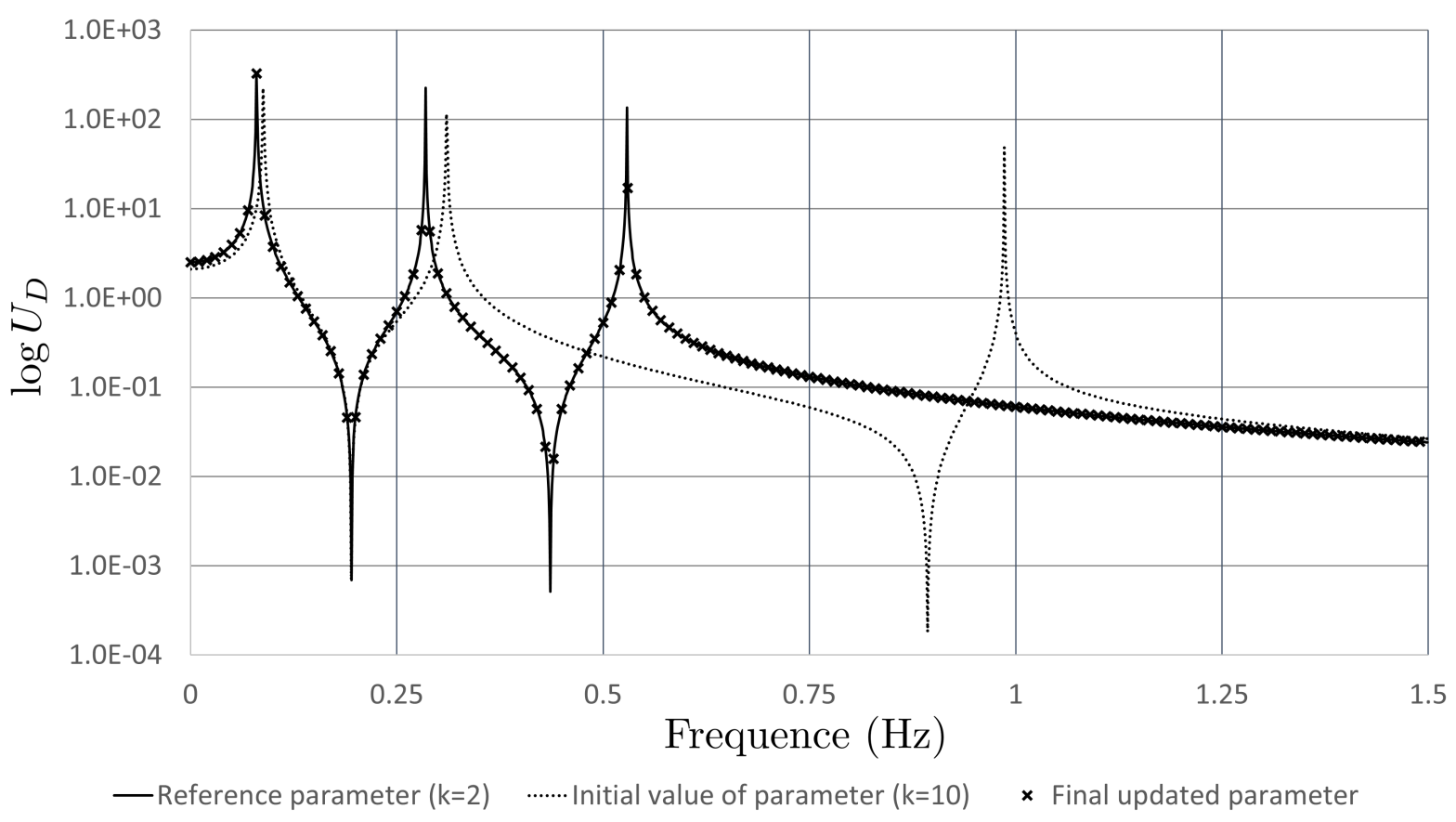

Figure 4: FRF at point D for different values of parameter $k$

So far, the reasoning has been based on the full set of measures, i.e., nodes B, C and D. However, in the real-world experiments the quantity of sensors are limited. In general, the number of sensors is well below the number of nodes present in the finite element mesh. This fact brings us to the next question. Is it possible to identify the joint parameter disposing only of partial measures? To find the answer, some supplementary numerical tests were made according to Table 3 . The numerical test T1 corresponds to Figure 3, whereas the remaining results are shown in Figure 5. The parameter is identified in all cases letting clear the robustness of the proposed formulation. In the limit, only one modal reference is enough for identification because the problem is essentially linear and with only one parameter to be identified. It is worth bearing in mind that these inferences are plausible because the reference data is free of uncertainties.

\begin{tabular}{cccc}
\hline Test & Reference eigenmode & Reference eigenfrequency & Measure at node \\
\hline T1 & $1,2,3$ & $1,2,3$ & B, C, D \\
T2 & 2 & 2 & B, C, D \\
T3 & 3 & 3 & B, C, D \\
T4 & 2 & 2 & D \\
T5 & 3 & 3 & B \\
\hline
\end{tabular}

Table 3: Numerical test details 


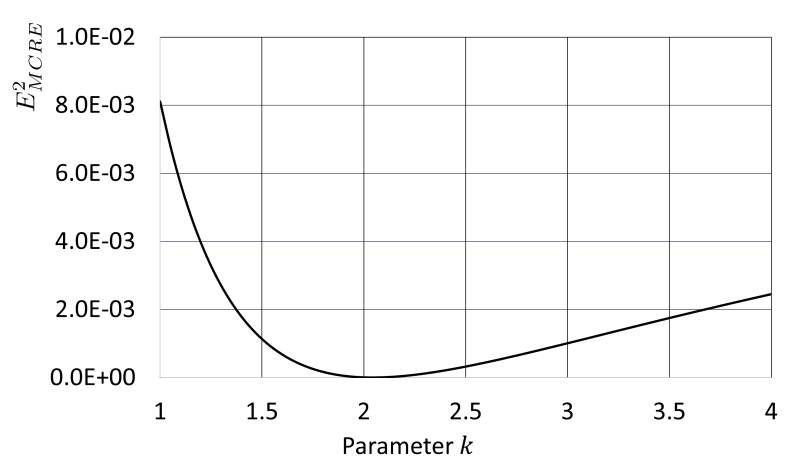

(a)

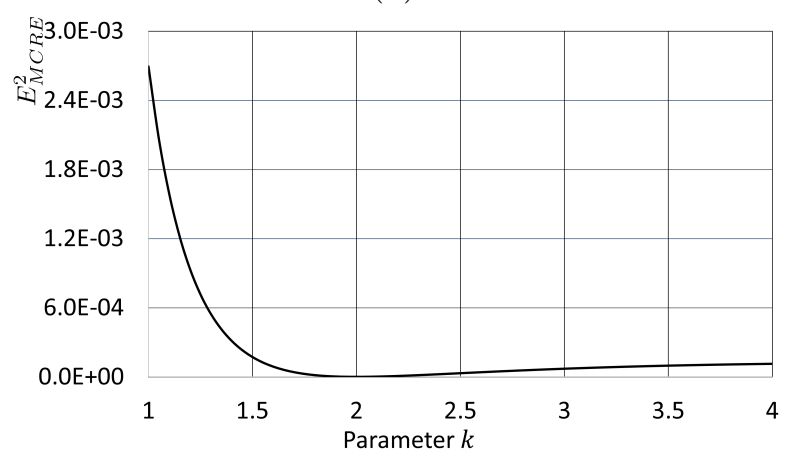

(c)

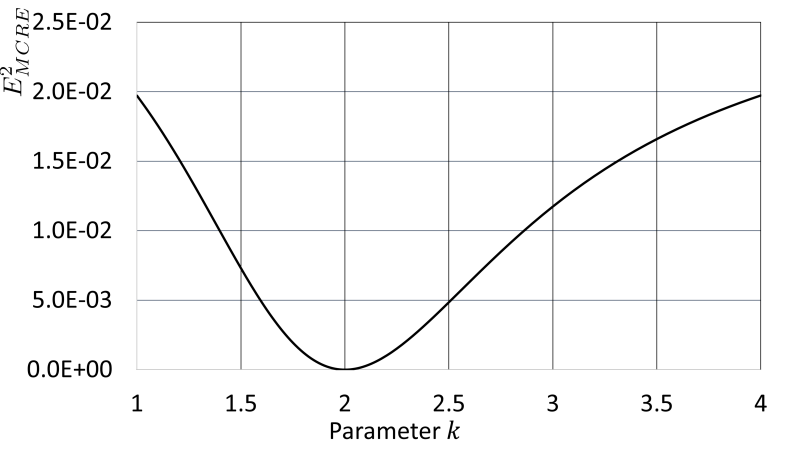

(b)

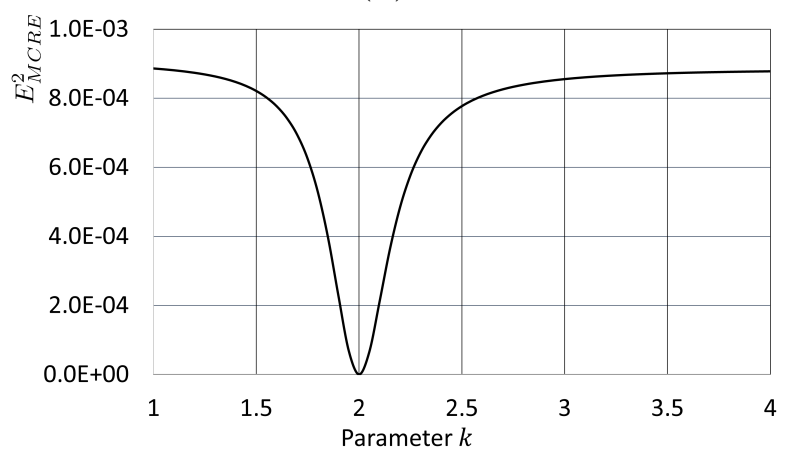

(d)

Figure 5: Norm MCRE as function of the design parameter for different testing cases.

(a) Test T2 (b) Test T3 (c) Test T4 (d) Test T5

The last aspect to be discussed in this example concerns the boundary conditions. In real world problems, perfectly rigid support conditions are hardly achieved. To mimic this situation, we propose to include an additional semi-rigid connection in place of the fixed support, i.e., at the node $A$. Then, we make its stiffness successively increase until it reaches very large values (virtually rigid) according to Table 4 . The goal is verifying whether the formulation can still identify both joints properly. In this case, the parametric space is two dimensional, $\left(k_{A}, k_{\text {joint }}\right)$, and a typical graph can be seen in Figure 6 for the case $\mathrm{C} 1$ from Table 4. The results are shown in Figures 7 to 11 for each parameter. Although the rigidity at extremity A increases drastically surpassing the order of magnitude of the remaining variables, the proposed formulation is still capable to identify it. As in the previous cases, this observation must be made with caution that uncertainties are not being taken into account.

\begin{tabular}{ccc}
\hline Parameter set & $k_{A}$ & $k_{\text {joint }}$ \\
\hline C1 & 5 & 2 \\
C2 & 10 & 2 \\
C3 & 50 & 2 \\
C4 & 200 & 2 \\
C5 & 2000 & 2 \\
\hline
\end{tabular}

Table 4: Reference values used for stability testing 


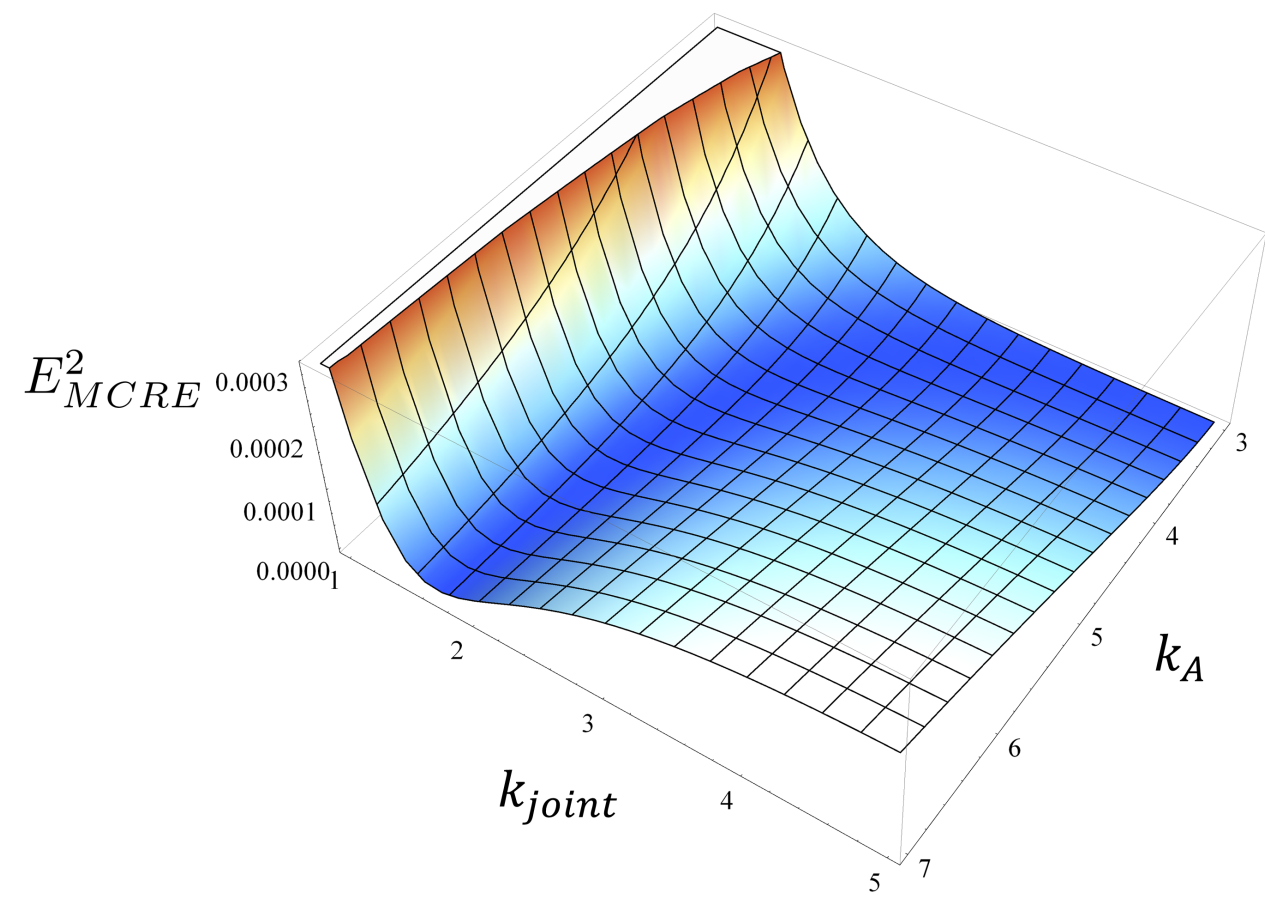

Figure 6: MCRE plotted in the neighbourhood of the minimum point for case C1

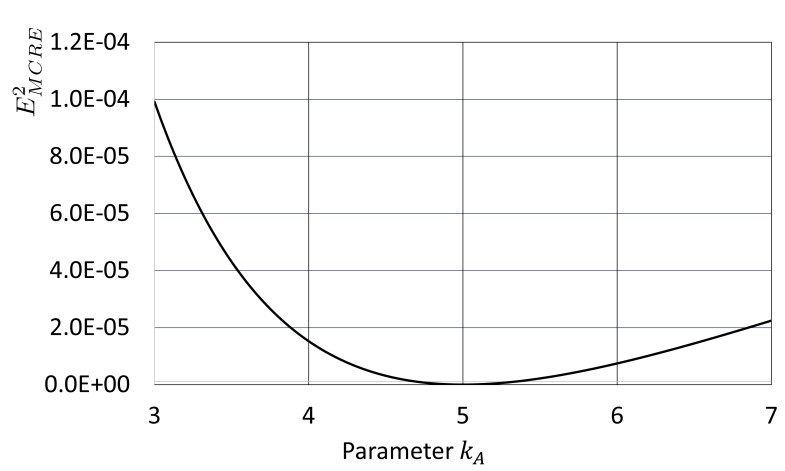

(a) C1: Parameter $k_{A}$

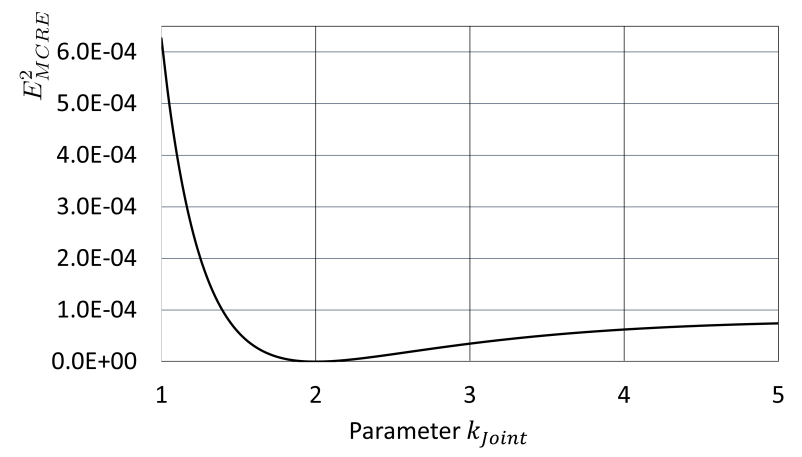

(b) C1: Parameter $k_{\text {joint }}$

Figure 7: Projection of the MCRE on each direction of the parameter domain. Case 1

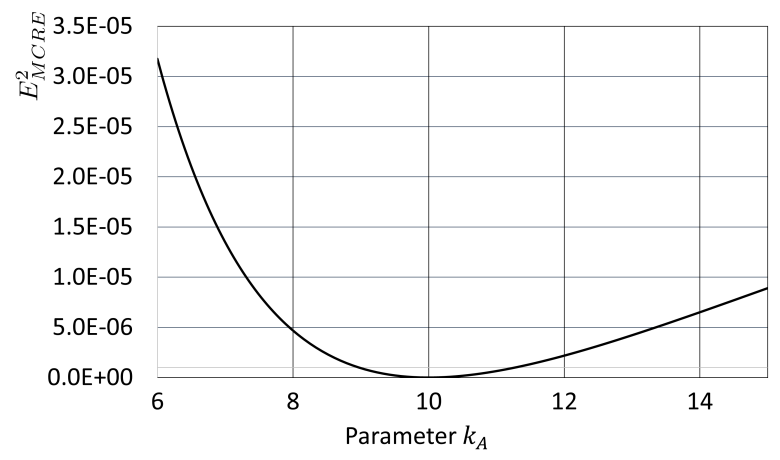

(a) C2: Parameter $k_{A}$

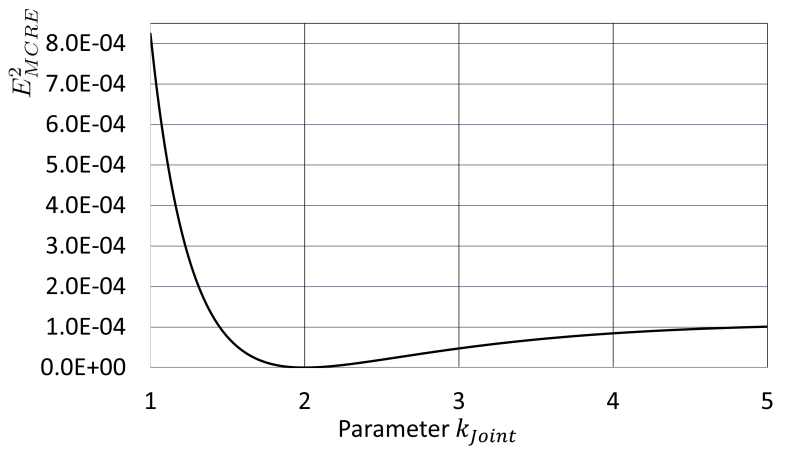

(b) C2: Parameter $k_{\text {joint }}$

Figure 8: Projection of the MCRE on each direction of the parameter domain. Case 2 


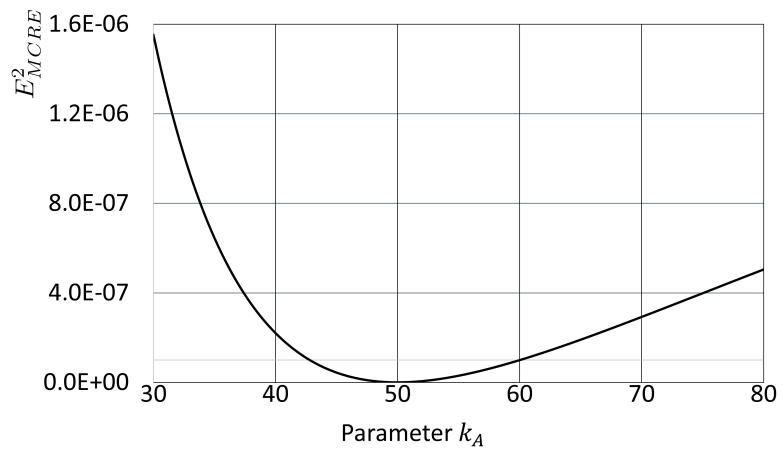

(a) C3: Parameter $k_{A}$

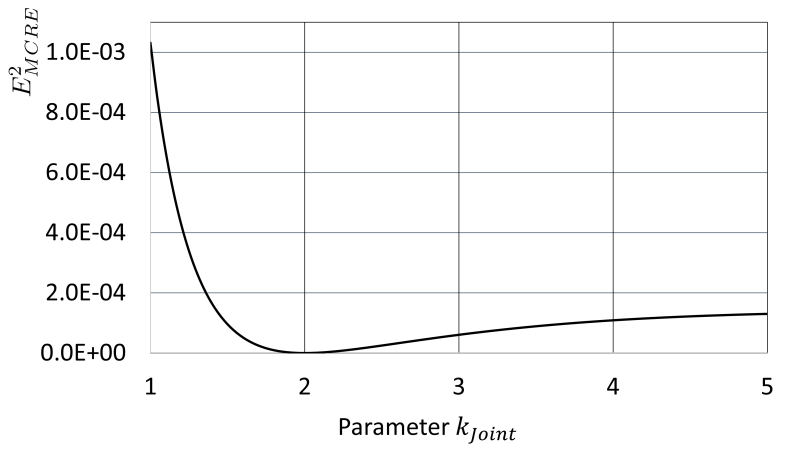

(b) C3: Parameter $k_{\text {joint }}$

Figure 9: Projection of the MCRE on each direction of the parameter domain. Case 3

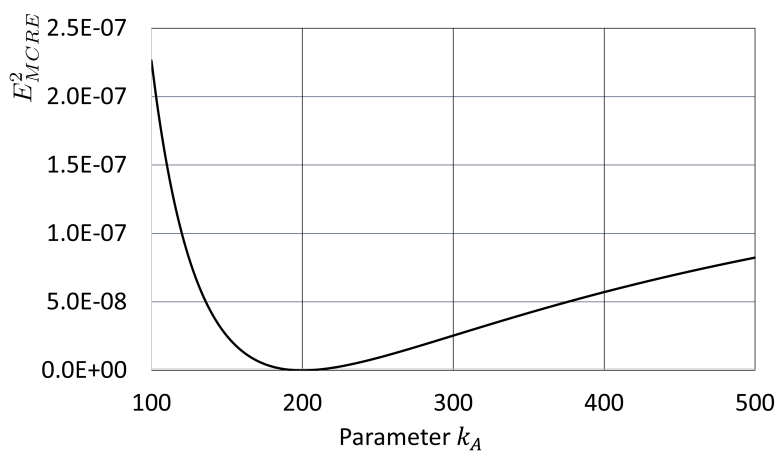

(a) C4: Parameter $k_{A}$

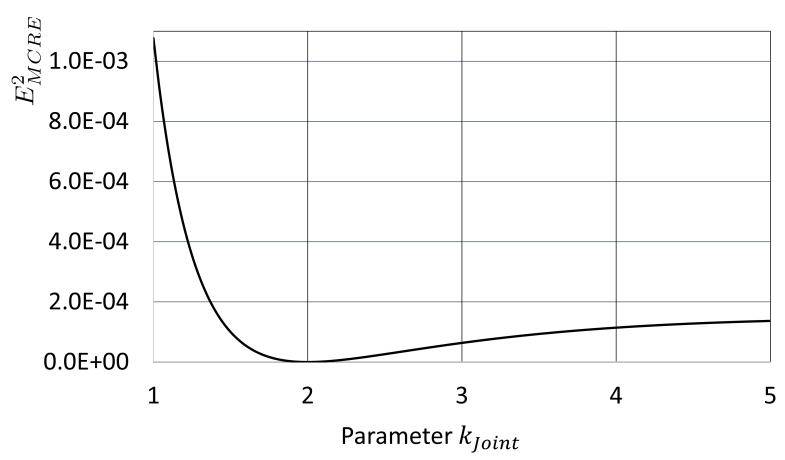

(b) C4: Parameter $k_{\text {joint }}$

Figure 10: Projection of the MCRE on each direction of the parameter domain. Case 4

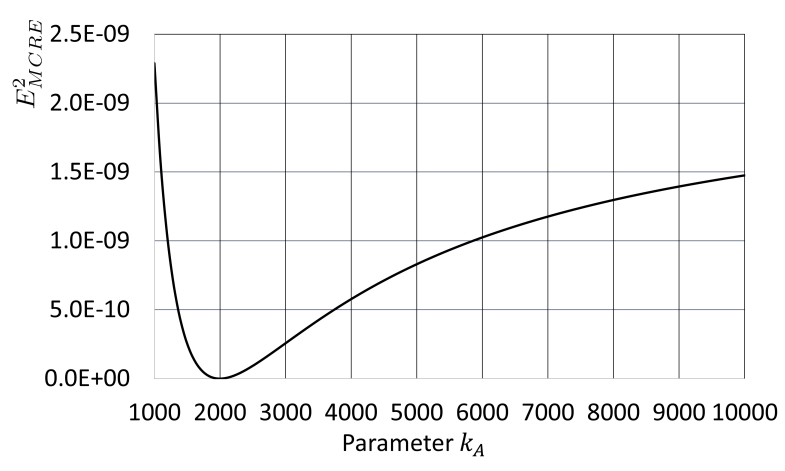

(a) C5: Parameter $k_{A}$

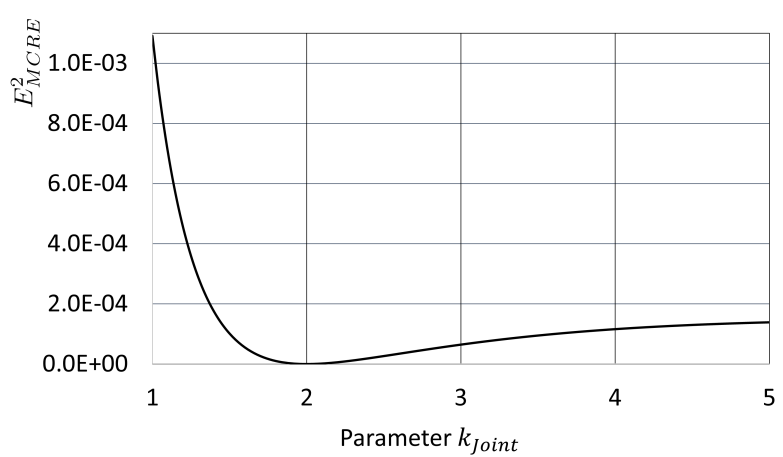

(b) C5: Parameter $k_{\text {joint }}$

Figure 11: Projection of the MCRE on each direction of the parameter domain. Case 5

The inverse problems are well known for being ill-posed, and consequently present difficulties in obtaining a numerical solution. However, the results obtained through this simple example show the attractiveness of the proposed methodology in deterministic contexts, in particular, the effectiveness with which the expected parameters are identified. This feature was maintained under different identification conditions such as complete reference measurement data, partial reference measurement data and semi-rigid boundary 
conditions. These inferences allow the continuation of numerical investigations retaining that, under well controlled conditions, numerical instabilities do not seem to represent major problems.

\subsection{Beam structure}

The present example was proposed by Friswell and Mottershead [14]. It is a beam with flexural rigidity $E I=4557 \mathrm{Nm}^{2}$, Poisson's ratio $\nu=0.3$, mass density $\rho=2680 \mathrm{Kg} / \mathrm{m}^{3}$ and length $L=0.7 \mathrm{~m}$. The schematic representation can be found in Figure 12 . The reference values are $k_{l}=4 \times 10^{7} \mathrm{~N} / \mathrm{m}$ and $k_{t}=1 \times 10^{5} \mathrm{~N} \cdot \mathrm{m} / \mathrm{rad}$.

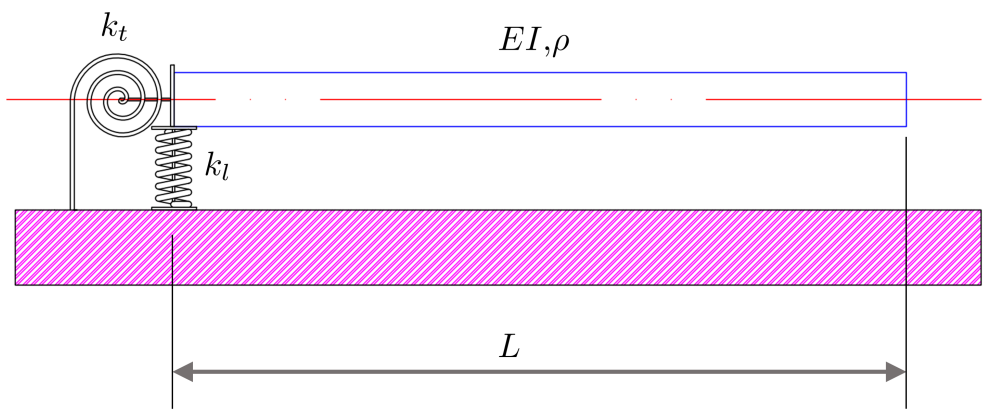

Figure 12: Beam schematic representation

The plot of the MCRE along the search interval can be seen in Figure 13. The identification was based on the first three vibration modes and corresponding eigenfrequencies. The measures (displacement and rotation) were taken only at the free end of the beam. As can be noted, the minimum point corresponds exactly to the reference value.

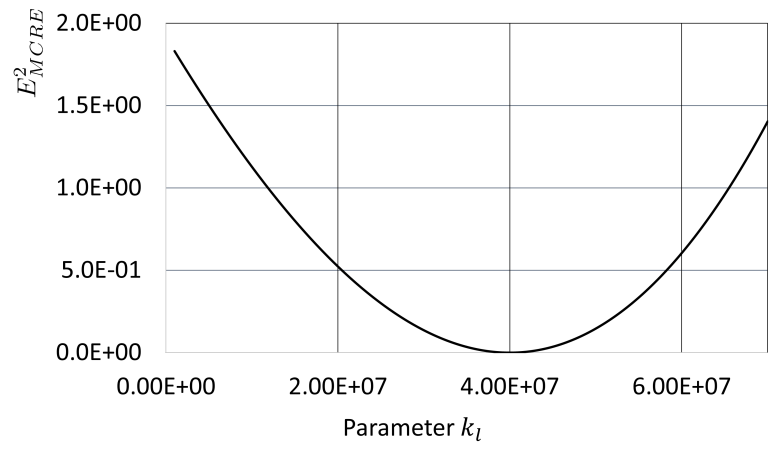

(a) Translational rigidity

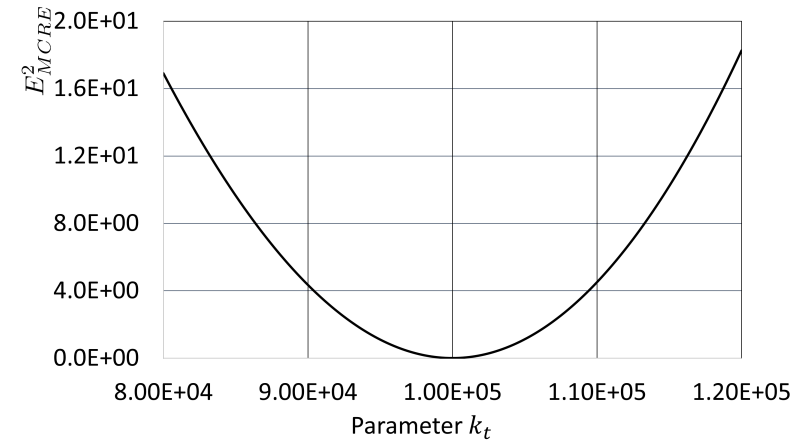

(b) Rotational rigidity

Figure 13: MCRE based identification

The measure presented in equation (38) has been consolidated in the literature for model identification purposes. So, we decided to apply it here and check if the predicted parameters remains the same. The parameter $h_{i}$ is set to 0.5 . The results are shown in Figure 14 retaining the same search interval. As can be seen, the classical MAC approach recovers the same values identified by the MCRE norm. 


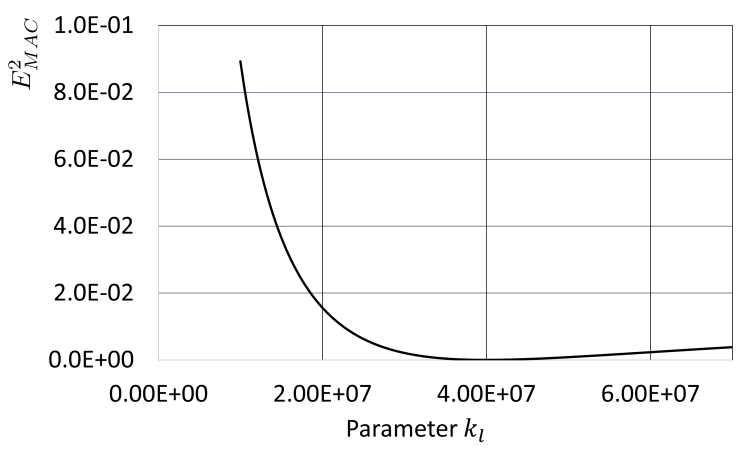

(a) Translational rigidity

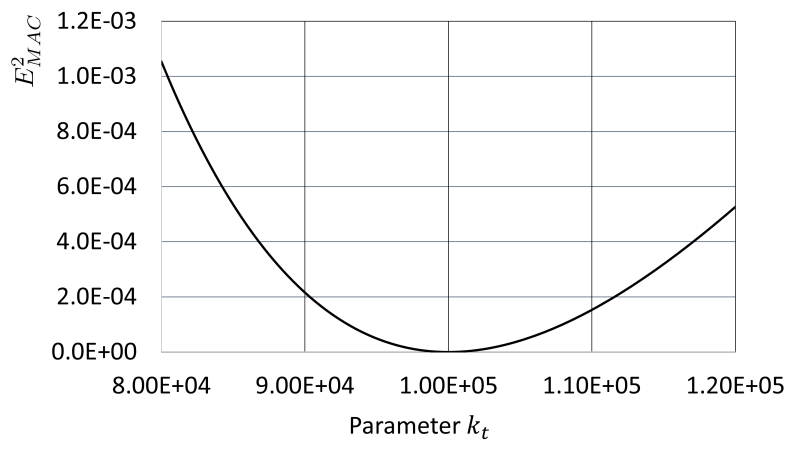

(b) Rotational rigidity

Figure 14: MAC based identification

From Figure 13-a and Figure 14-a, comparing MCRE norm and MAC norm, it can be noted that the MAC norm may exhibit less pronounced convexity in the vicinity of the minimum point. This observation serves as a warning when algorithms present convergence difficulties. Additionally, it indicates that the MCRE norm may be advantageous in some situations by preserving its parabolic aspect near the point of identification.

The relevance of this example lies in the fact that the proposed MCRE norm reproduces, with high degree of agreement, the results predicted by the classic MAC norm. This finding is encouraging from a theoretical point of view and many numerical analysis may follow from this. Despite its importance, this aspect will not be further explored.

These two examples explored exclusively deterministic aspects of the proposed formulation. This information is valuable for the theoretical development of new applications and extensions of the concepts discussed in this paper. However, in realistic situations, it is necessary to remember that determinism has limitations. For this reason, in the next example, this aspect is placed under discussion.

\subsection{Building structure}

This example refers to a building structure. Among the various types of structural design possible, the present has the particularity of being composed of planar elements. Thus, their carrying capacity is determined by the proper performance of slabs, walls and joints. Membrane effects are considered relevant in this case, so shell elements are utilized. The main input parameters are showed in table 5 whereas the front and the side view are presented in Figure 15. Each connection between a slab and the neighbouring wall is semi-rigid. The stiffness reference value is $4 \times 10^{6} \mathrm{~N} . \mathrm{m} / \mathrm{rad}$. 


\begin{tabular}{cc}
\hline Characteristic & Value \\
\hline Length $L_{X}$ & $14 \mathrm{~m}$ \\
Length $L_{Y}$ & $7 \mathrm{~m}$ \\
Length $L_{Z}$ & $21 \mathrm{~m}$ \\
Interfloor distance & $3 \mathrm{~m}$ \\
Wall thickness & $20 \mathrm{~cm}$ \\
Slab thickness & $14 \mathrm{~cm}$ \\
Young Modulus & $32 \mathrm{GPa}$ \\
Poisson's ratio & 0.2 \\
Specific weight & $2500 \mathrm{~kg} / \mathrm{m}^{3}$ \\
\hline
\end{tabular}

Table 5: Building structure details
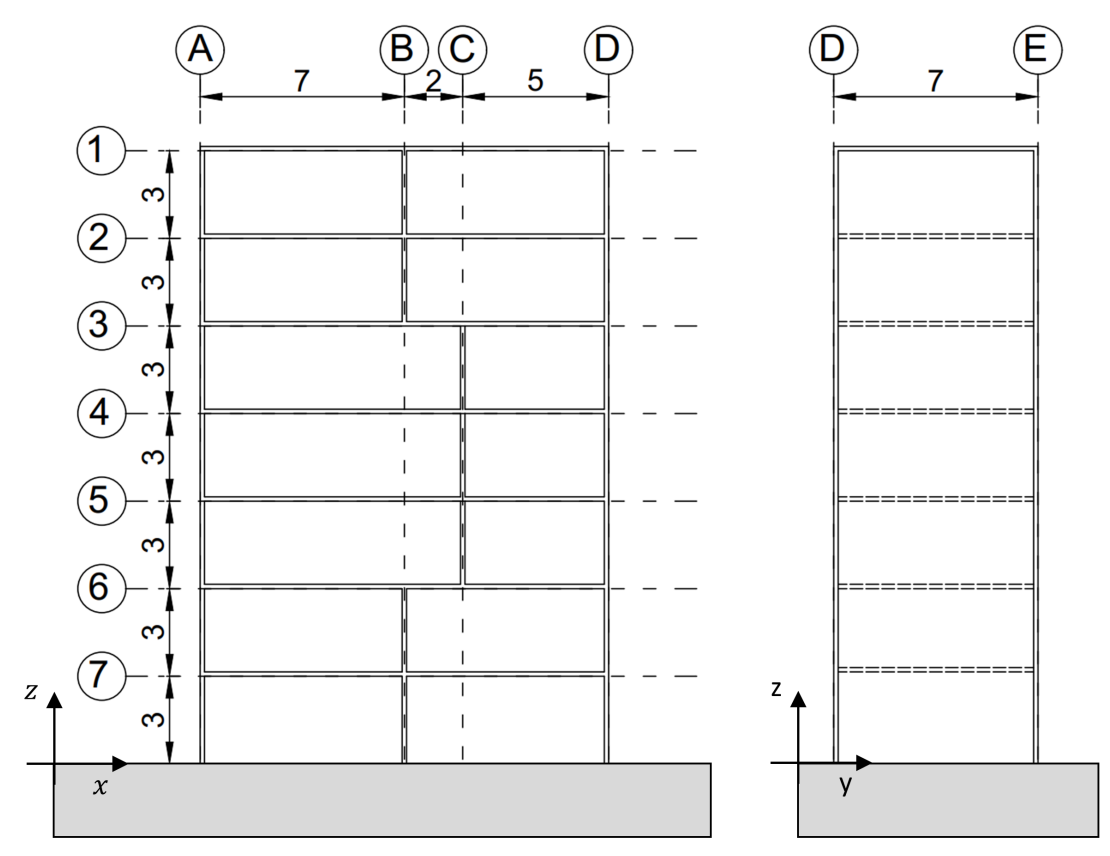

Figure 15: Building structure: Front and side view (measure in $m$ )

Only the first four modes will be considered for the joint identification. These are the reference modes with corresponding frequencies that can be seen in Figure 16. It will be considered the data from four arbitrary points (four sensors). They are chosen as being the four top vertex of the building. The measures are taken along the three displacement axis. Rotations can be included, but they were neglected in this application. It mimics the real measurement cases where the information is available for a limited set of points along particular directions.

Following the discussions presented in the previous examples, the identification, without considering the associated uncertainties, can be found in Figure 17. All observations made in those simpler examples remain valid here and will not be rediscussed. Nevertheless, a further comment is pertinent. Since the proposed formulation is built based on the FEM, it inherits the vast range of applications provided by this method. Not only 
reticular and planar structures can be analysed but also solid, and mixed, depending on the assembling capacity of the software that will host the formulation. The Cast3m [43] finite element code, developed in the present study, can handle these assemblies without major difficulties.

Continuing the numerical investigations, the non-determinism is included following three steps. In the first step, we will evaluate only the effects that measurement noise can cause. In the second stage, only the uncertainties regarding the material parameters will be addressed. In the last stage, we will evaluate both effects simultaneously. This progressive way of acting facilitates the analysis and interpretation of the results.

Moving on to the first step, noises are included in the reference measures. The measurement provided by each sensor follows a probabilistic distribution law assumed as Gaussian. The mean always corresponds to the corresponding deterministic value, and the standard deviation is adopted as a fraction of the mean value. Four percentile levels were tested: $5 \%, 10 \%, 15 \%$ and $20 \%$. One assumes that the more dispersed the distribution, the higher the noise level that affects the sensor and consequently the identification process. The results are shown in Figure 18 and Table 6. As the noise level increases the MCRE evolution paths become horizontal. The more horizontal the curves, the more the algorithm loses its identification capability. This is because the identified parameter corresponds to a minimum point which is in turn found by a gradient method. The more horizontal the curve, the harder it becomes to define the gradient, so the algorithm starts to get too slow. Excessively long calculation times may become prohibitive in practical applications. In the numerical tests, below the maximum noise level (20\%), the proposed formulation was still able to identify properly the rotational stiffness of the joints. 


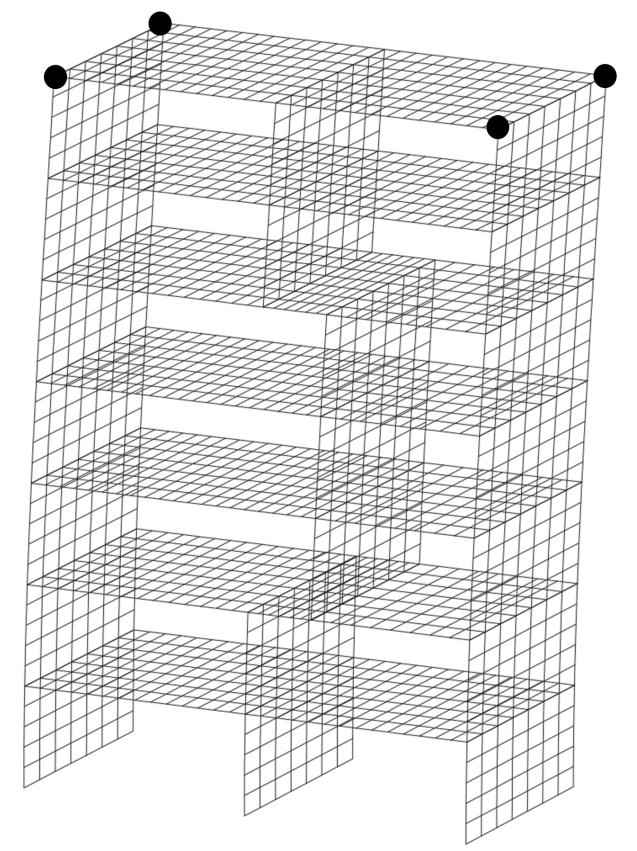

(a) Mode $1, f_{1}=0.47 \mathrm{~Hz}$

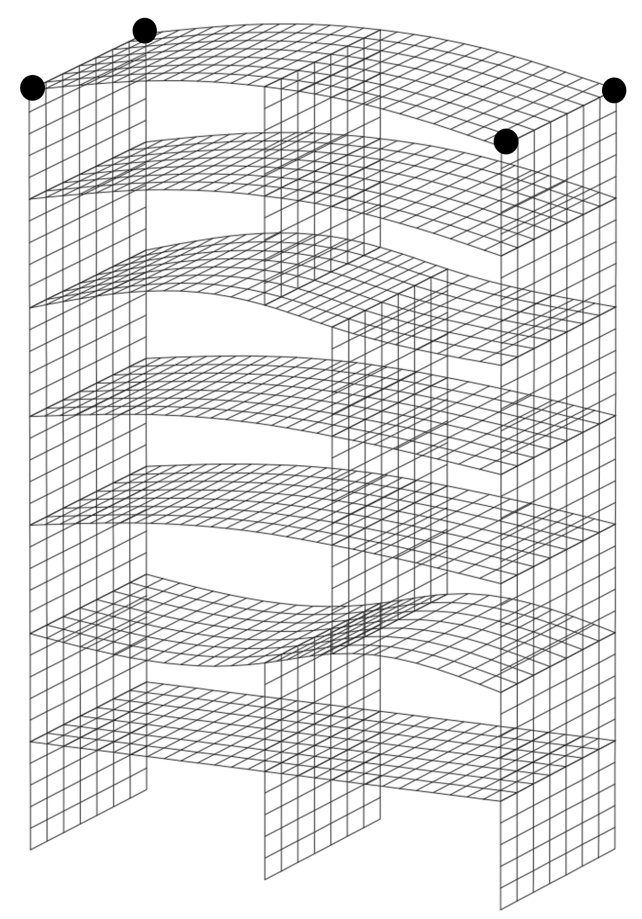

(c) Mode $3, f_{3}=2.46 \mathrm{~Hz}$

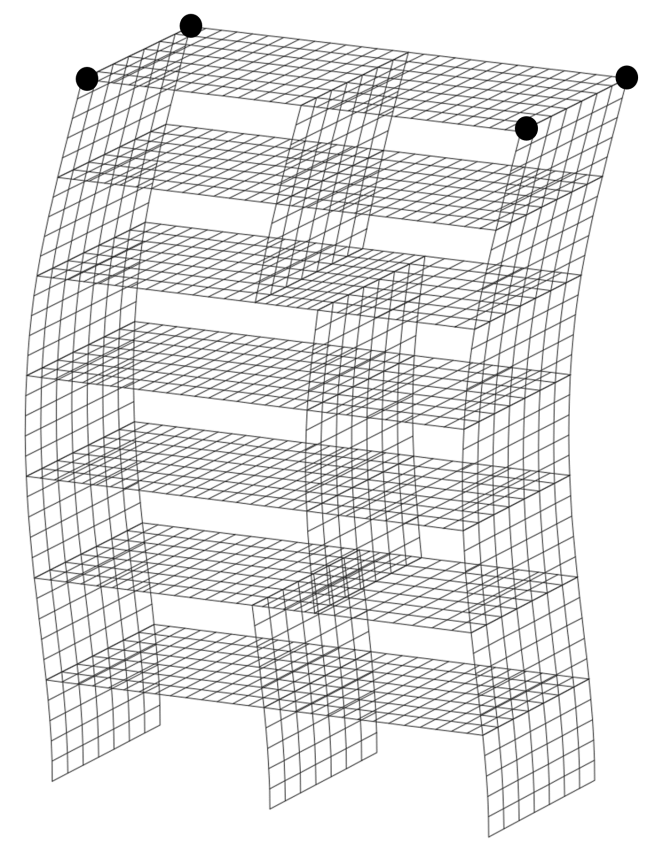

(b) Mode $2, f_{2}=1.66 \mathrm{~Hz}$

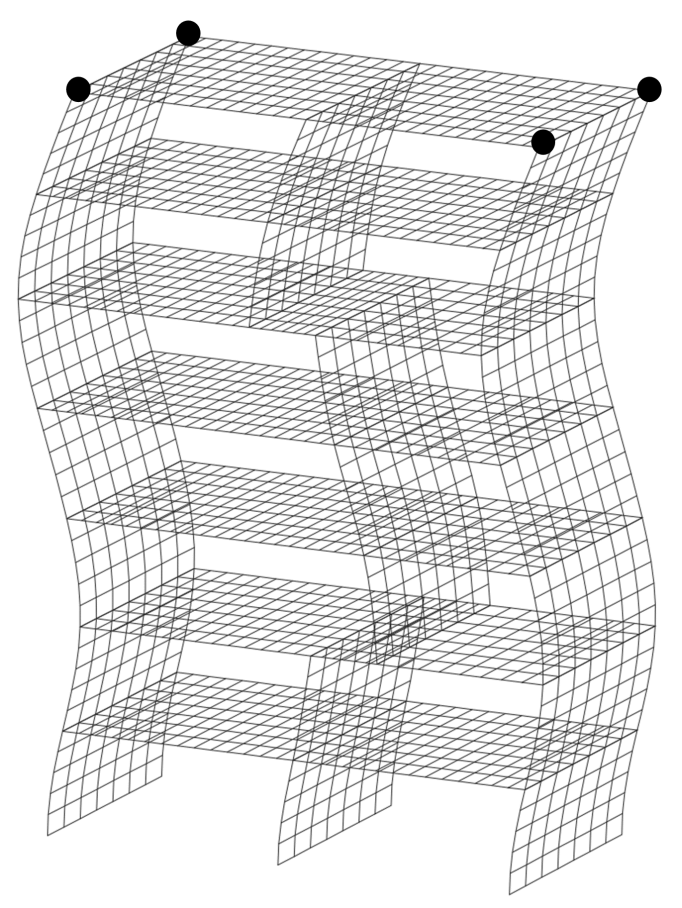

(d) Mode 4, $f_{4}=3.38 \mathrm{~Hz}$

Figure 16: Building reference mode shapes. Small bullets indicate sensor positions

Proceeding to the second step, it is evaluated how uncertainties may influence the results. Recalling that noise effects are not considered at this stage, the methodology follows what was described in Section 2.5. Three variables are then investigated: the Young modulus, Poisson's ratio and the specific weight. Each of these input parameters is considered as a Gaussian random variable with mean values given by Table 5 and a standard deviation of $20 \%$ of the mean. After defining the response polynomial, $10^{5}$ sets 


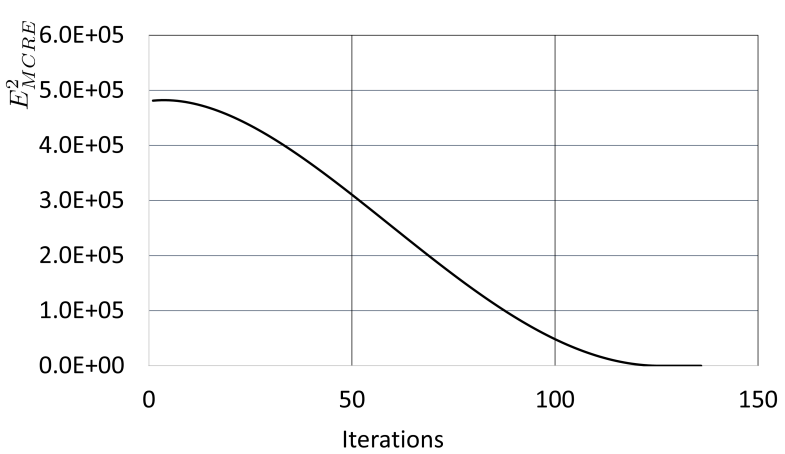

(a)

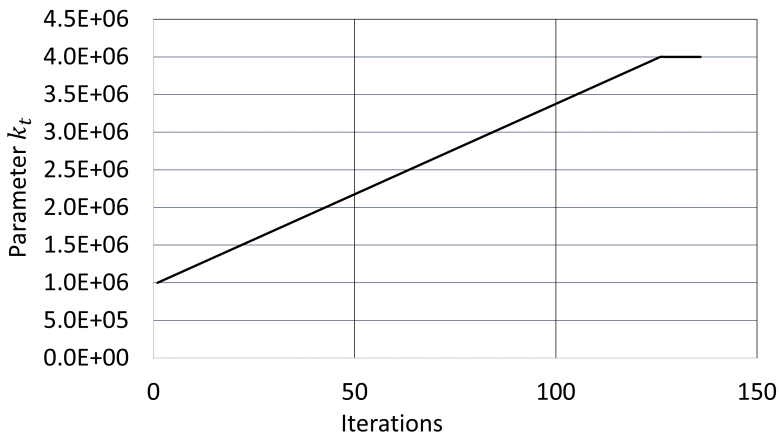

(b)

Figure 17: Fully deterministic identification (a) Modified Constitutive Relation Error evolution (b) Rotational rigidity evolution

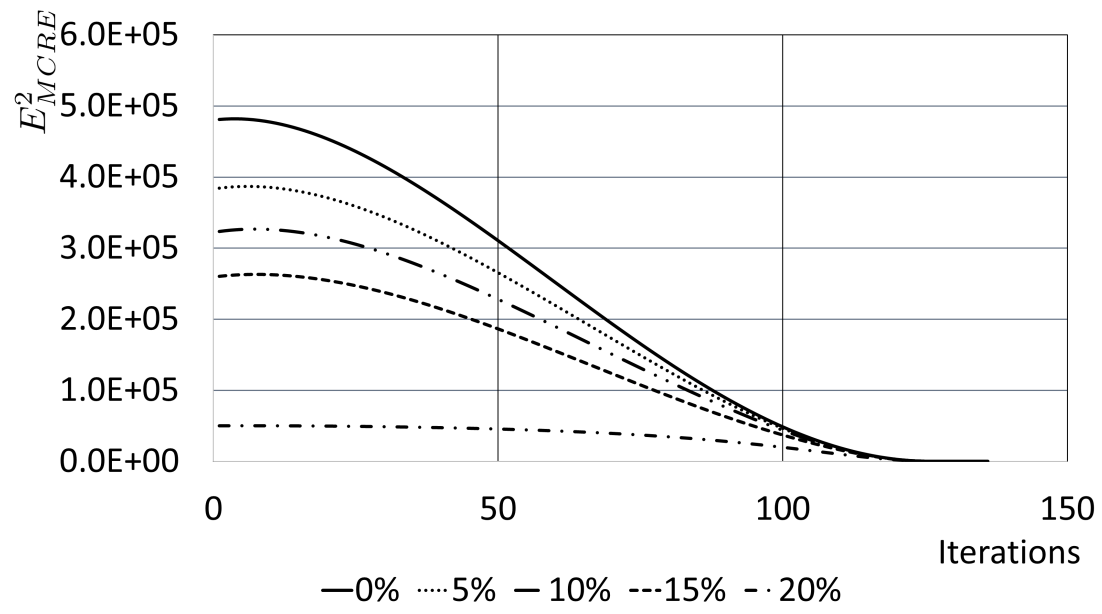

Figure 18: MCRE evolution in the presence of increasing noise levels

\begin{tabular}{cc}
\hline Noise level & Identified parameter $k_{t}$ \\
\hline $0 \%$ & $4.00 \times 10^{6} \mathrm{~N} . \mathrm{m} / \mathrm{rad}$ \\
$5 \%$ & $4.05 \times 10^{6} \mathrm{~N} . \mathrm{m} / \mathrm{rad}$ \\
$10 \%$ & $3.97 \times 10^{6} \mathrm{~N} . \mathrm{m} / \mathrm{rad}$ \\
$15 \%$ & $3.95 \times 10^{6} \mathrm{~N} . \mathrm{m} / \mathrm{rad}$ \\
$20 \%$ & $3.92 \times 10^{6} \mathrm{~N} . \mathrm{m} / \mathrm{rad}$ \\
\hline
\end{tabular}

Table 6: Identified $k_{t}$ in the presence of increasing noise levels 


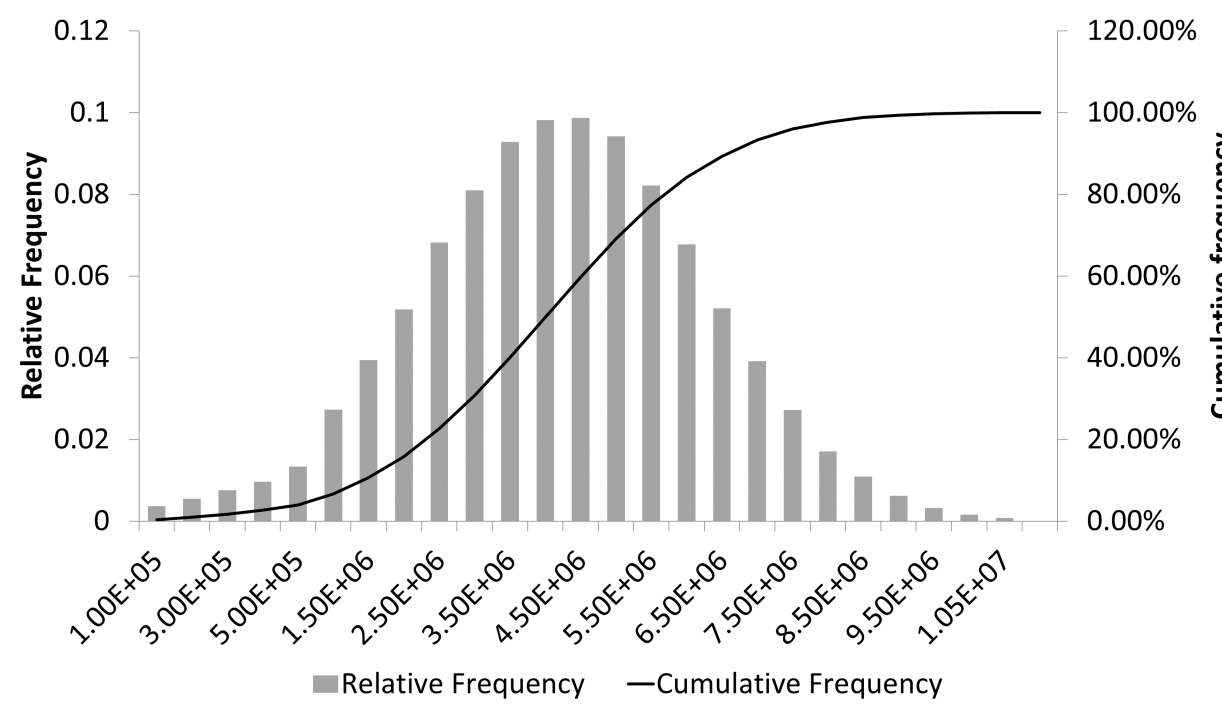

Figure 19: Discrete probability density of the rigidity identified considering the uncertainties on material parameters without noise

of parameters are drawn and tested to verify the value of the predicted joint stiffness. The discrete spectrum of identified parameters can be seen in Figure 19. This graph shows that rotational rigidity dispersion resembles a Gaussian law. The calculation of the mean and standard deviation gives the values $4.023 \times 10^{6} \mathrm{~N} . \mathrm{m} / \mathrm{rad}$ and $1.953 \times 10^{6} \mathrm{~N} . \mathrm{m} / \mathrm{rad}$, respectively. The mean of the identified values tends to the reference value, while the deviation reaches $\sim 50 \%$. In this case it can be inferred that the identification algorithm may increase the dispersion without changing their mean value w.r.t. the deterministic. This behaviour is typical of linear correlations between random variables. Thus, the proposed identification algorithm manifests a linear dependence with respect to the material uncertainties. This feature is relevant because it suggests that the quality control of the identification process can be performed by controlling the dispersion of the referred input variables. However, deeper statistical studies are needed to identify the validity limits of this statement, which is beyond the scope of the present study.

It is now pertinent to find out what happens when all uncertainties are simultaneously included into the model, i.e. the third stage. As the uncertainties associated with the material do not impact significantly the identification capability, they will be maintained at the levels already presented, i.e. 20\%. On the other hand, the measures provided by the sensors are considered have increasing noise levels: 1\%,5\%, and 10\%. After running $10^{5}$ Monte Carlo simulations, the dispersion corresponding to each set may be expressed graphically as shown in Figures 20, 21 and 22. As the noise level in the measurements increases, the dispersion curve changes its appearance from normal to log-normal. The rising peaks on the left side of the graphs indicate that an increasing number of observations are moving away from the mean. This fact is confirmed by the calculated values, which are: $3.92 \times 10^{6} \mathrm{~N} . \mathrm{m} / \mathrm{rad}($ noise $=1 \%), 2.83 \times 10^{6} \mathrm{~N} . \mathrm{m} / \mathrm{rad}($ noise $=5 \%)$ and $2.28 \times 10^{6} \mathrm{~N} . \mathrm{m} / \mathrm{rad}$ (noise $=10 \%$ ). The presence of material uncertainty seems to amplify the effects of noise levels, which ultimately create difficulties for the identification process. 


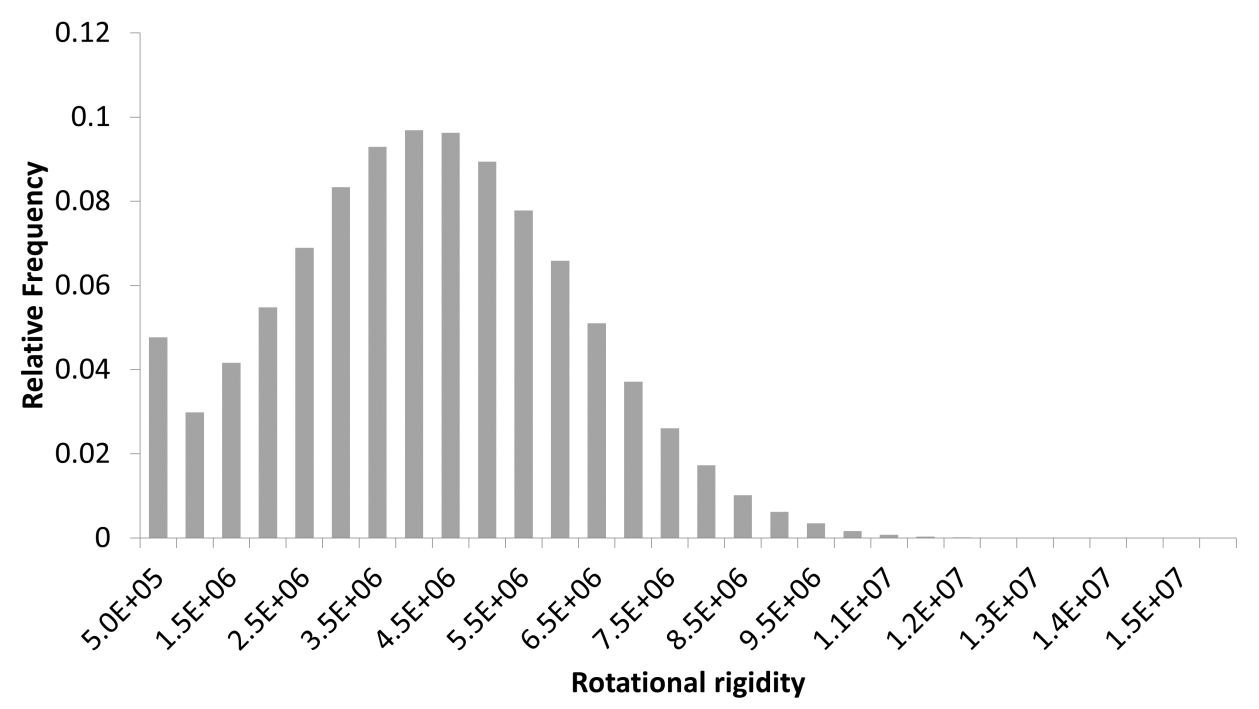

Figure 20: Discrete probability density of the rigidity identified considering the uncertainties on material parameters in the presence of noise (level $=1 \%$ )

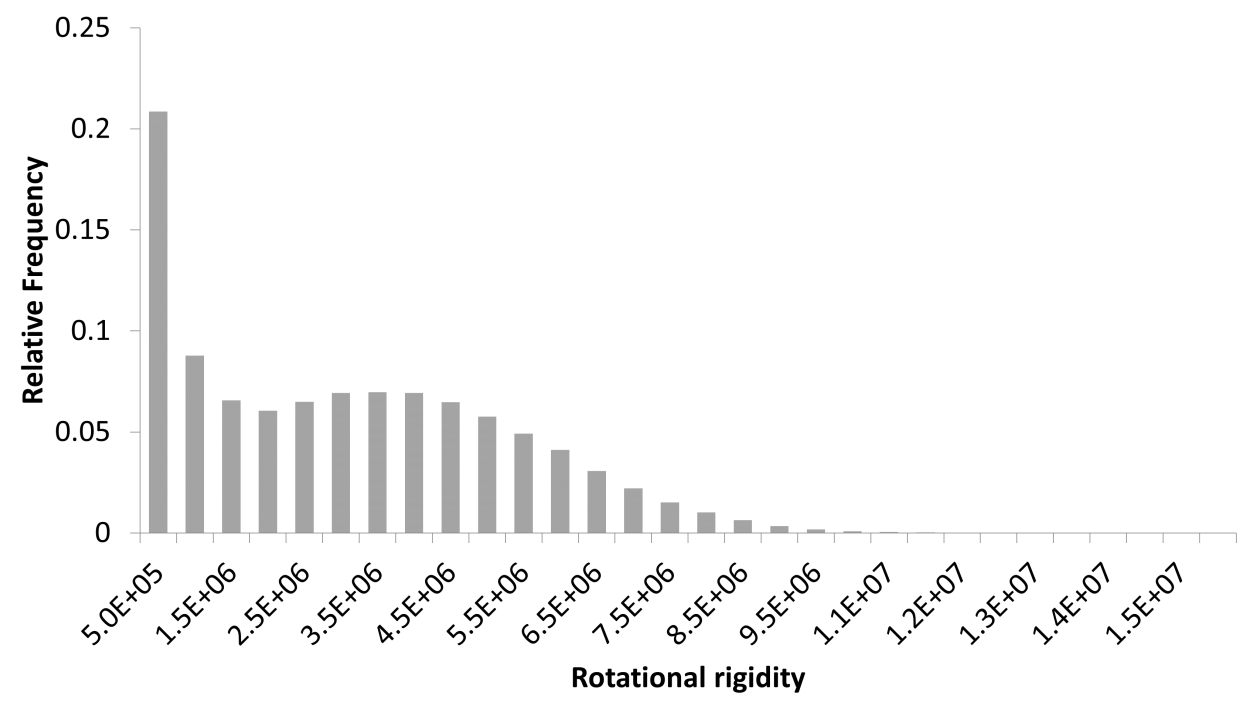

Figure 21: Discrete probability density of the rigidity identified considering the uncertainties on material parameters in the presence of noise (level $=5 \%)$ 


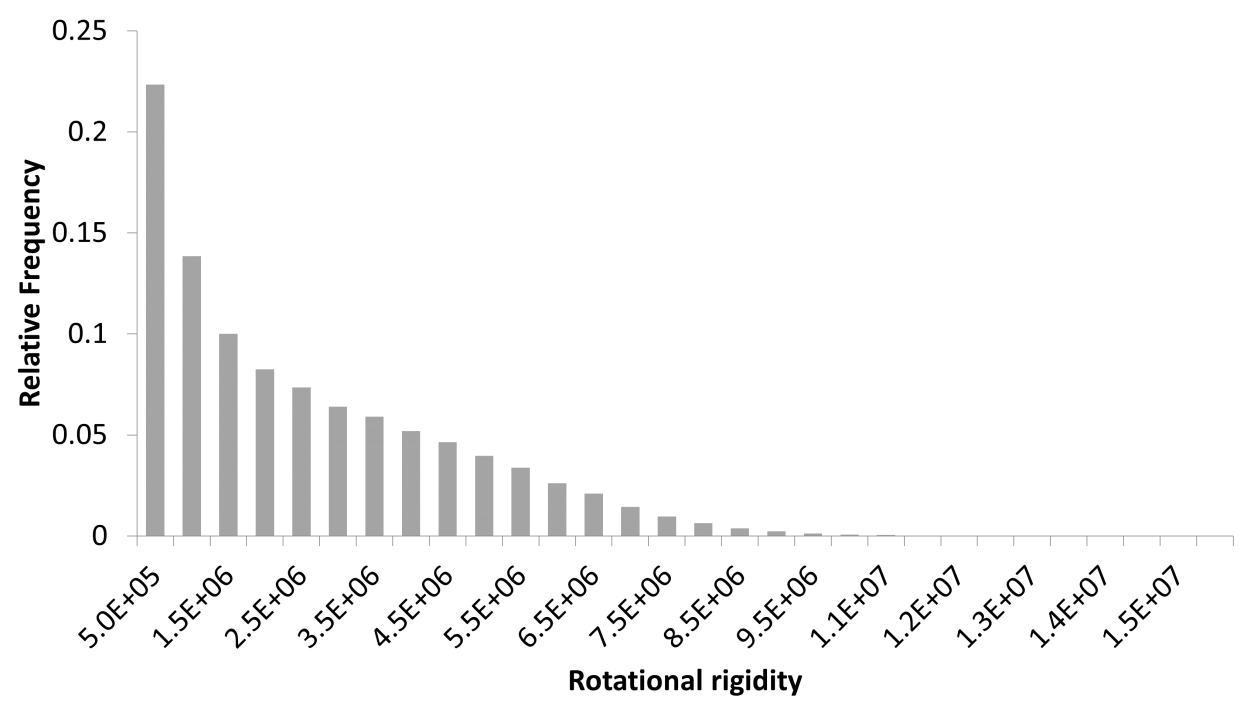

Figure 22: Discrete probability density of the rigidity identified considering the uncertainties on material parameters in the presence of noise (level $=10 \%)$

The outcome of these three steps is that the quality of the measures may influence directly the identification ability of the proposed formulation. Although a certain robustness against some categories of uncertainty, such as materials for instance, can be evidenced, it rests not immune to high noise levels.

\section{$4 \quad$ Final remarks}

The present study proposed a numerical formulation for the parameter identification of joints between structural elements. Joints are considered as independent mechanical entities that are capable of transmitting generalized forces between their neighbouring structural elements. It is assumed that they have a priori unknown rigidity, but can be identified from information about the free vibration regime of the global structure. The numerical procedure follows the precepts of the Constitutive Relation Error theory, in which the unknown stiffness parameters are found through a minimization problem. Although planar elements are the focus of the present study, the technique can also be applied to elements of other typologies.

The main characteristics and potentialities were evidenced numerically by examples of increasing complexity, starting with reticular structures and progressing to planar structures. In particular, this investigation highlights that the proposed algorithm inherits the versatility of using FEM in combining different types of structural elements, as long as the host software has the corresponding assembling capability. This feature opens numerous research opportunities and applications in engineering.

In numerical identification procedures, the sources of uncertainty occupy a prominent place. Thus, the discussions throughout the text followed two complementary paths. The first was to investigate the predictability of the proposed formulation in a completely deterministic context. As expected, its performance was remarkable. It has been shown that in ideal cases (where all input variables are perfectly known and free of noise), the formulation can identify joint parameters properly even with partial information. Although 
these observations are of significant theoretical interest, the assumed determinism has limitations in demonstrating the applicability of the formulation to more general cases.

The second path was to model the inherent uncertainties coming from various sources. The idea was checking if the good deterministic properties remained. To this end, it was included a probabilistic analyses by means of surface response metamodeling. This choice was made because probabilistic analyses require a large number of finite element model calls, which can quickly result in prohibitive processing times. The obtained results are encouraging. They show that the proposed formulation retains its ability to identify the joint parameter within limited levels of material uncertainty and noise. In the tests performed, the uncertainties associated exclusively with the material were not so harmful in the identification process. However, high noise levels in measurements may lead the proposed algorithm to lose its efficiency in properly predicting parameters.

The present study finds ramifications in different areas of engineering, such as the design of experiments. Using the proposed formulation, it is possible to establish the best geometric arrangement of sensors according to the parameter to be identified. Another application would be in the area of structural monitoring, where different monitoring devices can be proposed based on the concepts discussed here.

Some challenges still persist. Among them, it is cited the inclusion of more complex joint models, possibly non-linear. Thus, it would be possible to advance the proposed formulation in the study of damaged structural states and consequently a better estimation of its remaining resistant capacity.

\section{Acknowledgment}

This work, within the SINAPS@ project, benefited from French state funding managed by the National Research Agency under program RNSR Future Investments bearing reference No. ANR-11-RSNR-0022-04. The research reported in this paper has been supported in part by the SEISM Paris Saclay Research Institute.

\section{References}

[1] M. Petyt and W. Mirza. Dynamic behaviour of in-line shear walls connected by floor slabs. Journal of Sound and Vibration, 25(3):349-357, 1972.

[2] N. Lle and J. Reynouard. Seismic behavior of $\mathrm{r} / \mathrm{c}$ shear wall structures designed according to the french ps92 and ec 8 codes: A comparison between shaking-table response data and 2d modeling. In 12th World Conference on Earthquake Engineering, 2000.

[3] Ebrahim Zamani Beydokhty and Hashem Shariatmadar. Behavior of damaged exterior rc beam-column joints strengthened by cfrp composites. Latin American journal of solids and structures, 13(5):880-896, 2016.

[4] Alg Kudzys and Alg Kudzys. Evaluation of wall-slab connection behaviour under extreme lateral actions. Statyba, 2(8):35-44, 1996. 
[5] Snehal Kaushik and Kaustubh Dasgupta. Seismic behavior of slab-structural wall junction of rc building. Earthquake Engineering and Engineering Vibration, 18(2):331-349, 2019.

[6] S Greeshma and KP Jaya. Effect of geometric parameters on exterior wall-floor connection. In 15 WCEE, 2012.

[7] Johannes Daniël Gerber and GPAG van Zijl. Alternative wall-to-slab connection systems in reinforced concrete structures. Journal of the South African Institution of Civil Engineering, 59(3):36-47, 2017.

[8] Snehal Kaushik and Kaustubh Dasgupta. Seismic damage in shear wall-slab junction in rc buildings. Procedia Engineering, 144:1332-1339, 2016.

[9] GM Cocchi. Elastic-static analysis of shear wall/slab-frame systems using the framework method. Computers $\&$ structures, 54(2):303-313, 1995.

[10] Morris Riedel, Felix Wolf, Dieter Kranzlmüller, Achim Streit, and Thomas Lippert. Research advances by using interoperable e-science infrastructures. Cluster computing, 12(4):357, 2009.

[11] F. Hemez and S. Doebling. Review and assessment of model updating for non-linear, transient dynamics. Mechanical Systems and Signal Processing, 15(1):45-74, 2001.

[12] Z. Pan, S. Guner, and F. Vecchio. Modeling of interior beam-column joints for nonlinear analysis of reinforced concrete frames. Engineering Structures, 142:182$191,2017$.

[13] J. Mottershead and M. Friswell. Model updating in structural dynamics: a survey. Journal of sound and vibration, 167(2):347-375, 1993.

[14] M. Friswell and Mottershead J. Finite element model updating in structural dynamics, volume 38. Springer Science \& Business Media, 1995.

[15] B. Zárate and J. Caicedo. Finite element model updating: multiple alternatives. Engineering Structures, 30(12):3724-3730, 2008.

[16] Y.B. Yang and Y.J. Chen. A new direct method for updating structural models based on measured modal data. Engineering Structures, 31(1):32-42, 2009.

[17] J. Mottershead, M. Link, and M. Friswell. The sensitivity method in finite element model updating: a tutorial. Mechanical systems and signal processing, 25(7):22752296, 2011.

[18] P. Ladevèze and D. Leguillon. Error estimate procedure in the finite element method and applications. SIAM J. Numer. Anal., 20(3):485 - 509, 1983.

[19] P. Ladevèze, D. Nedjar, and M. Reynier. Updating of finite element models using vibration tests. AIAA Journal, 32(7):1485 - 1491, 1994. 
[20] A. Deraemaeker, P. Ladevèze, and S. Le Loch. Results obtained by the cre updating method using a plate model. Mechanical systems and signal processing, 17(1):47-54, 2003.

[21] A. Deraemaeker, P. Ladevèze, and P. Leconte. Reduced bases for model updating in structural dynamics based on constitutive relation error. Computer methods in applied mechanics and engineering, 191(21-22):2427-2444, 2002.

[22] V. Decouvreur, P. Bouillard, A. Deraemaeker, and P. Ladevèze. Updating 2d acoustic models with the constitutive relation error method. Journal of sound and vibration, 278(4-5):773-787, 2004.

[23] V. Decouvreur, P. Ladevèze, and P. Bouillard. Updating 3d acoustic models with the constitutive relation error method: A two-stage approach for absorbing material characterization. Journal of sound and vibration, 310(4-5):985-997, 2008.

[24] P. Charbonnel, P. Ladevèze, F. Louf, and C. Le Noac'h. A robust cre-based approach for model updating using in situ measurements. Computers 6 Structures, 129:63-73, 2013.

[25] M. Azzouna, P. Feissel, and P. Villon. Robust identification of elastic properties using the modified constitutive relation error. Computer Methods in Applied Mechanics and Engineering, 295:196-218, 2015.

[26] S. Guchhait and B. Banerjee. Material parameter identification in transient dynamics by error in constitutive equation approach. Procedia Engineering, 144:512-519, 2016.

[27] B. Banerjee, T. Walsh, W. Aquino, and M. Bonnet. Large scale parameter estimation problems in frequency-domain elastodynamics using an error in constitutive equation functional. Computer methods in applied mechanics and engineering, 253:60-72, 2013.

[28] S. Huang, P. Feissel, and P. Villon. Modified constitutive relation error: An identification framework dealing with the reliability of information. Computer Methods in Applied Mechanics and Engineering, 311:1-17, 2016.

[29] J. Waeytens and et al. Model updating techniques for damage detection in concrete beam using optical fiber strain measurement device. Engineering Structures, 129:210, 2016.

[30] O. Allix, P. Feissel, and H. Nguyen. Identification strategy in the presence of corrupted measurements. Engineering computations, 22(5/6):487-504, 2005.

[31] P. Feissel and O. Allix. Modified constitutive relation error identification strategy for transient dynamics with corrupted data: The elastic case. Computer methods in applied mechanics and engineering, 196(13-16):1968-1983, 2007.

[32] J. Guo and I. Takewaki. Minimum constitutive relation error based static identification of beams using force method. In Journal of Physics: Conference Series, volume 842, pages 1-10. IOP Publishing, 2017. 
[33] T. Silva and N. Maia. Detection and localisation of structural damage based on the error in the constitutive relations in dynamics. Applied Mathematical Modelling, 46:736-749, 2017.

[34] Jia Guo, Li Wang, and Izuru Takewaki. Modal-based structural damage identification by minimum constitutive relation error and sparse regularization. Structural Control and Health Monitoring, 25(12):e2255, 2018.

[35] Jia Guo, Li Wang, and Izuru Takewaki. Frequency response-based damage identification in frames by minimum constitutive relation error and sparse regularization. Journal of Sound and Vibration, 443:270-292, 2019.

[36] Jia Guo, Kailai Deng, Li Wang, and Izuru Takewaki. Physical-based parametrization and local damage identification for frame-type structures using response sensitivity approach in time domain. Structural Control and Health Monitoring, 26(10):e2412, 2019.

[37] Jia Guo, Li Wang, and Izuru Takewaki. Static damage identification in beams by minimum constitutive relation error. Inverse Problems in Science and Engineering, 27(10):1347-1371, 2019.

[38] S. Guchhait and B. Banerjee. Constitutive error based parameter estimation technique for plate structures using free vibration signatures. Journal of Sound and Vibration, 419:302-317, 2018.

[39] H. Oliveira, F. Louf, E. Hervé-Secourgeon, and F. Gatuingt. Wall-slab joint parameter identification of a reinforced concrete structure using possibly corrupted modal data. International Journal for Numerical and Analytical Methods in Geomechanics, 44(1):19-39, 2020.

[40] P. Ladevèze and J. Pelle. Mastering calculations in linear and nonlinear mechanics, volume 171. Springer, 2005.

[41] P. Ladeveze. A modelling error estimator for dynamic structural model updating. In Studies in Applied Mechanics, volume 47, pages 135-151. Elsevier, 1998.

[42] Olek C Zienkiewicz, Robert L Taylor, and Jian Z Zhu. The finite element method: its basis and fundamentals. Elsevier, 2005.

[43] Cast3m. CEA, Logiciel de calcul par éléments finis en mécanique des structures et des fluides, available at http://www-cast3m.cea.fr/index.php, 2018.

[44] George EP Box and Kenneth B Wilson. On the experimental attainment of optimum conditions. Journal of the Royal Statistical Society: Series B (Methodological), 13(1):1-38, 1951.

[45] Christian G Bucher and Ulrich Bourgund. A fast and efficient response surface approach for structural reliability problems. Structural safety, 7(1):57-66, 1990. 